\title{
EXPANSIONS FOR THE DISTRIBUTION OF $M$-ESTIMATES WITH APPLICATIONS TO THE MULTI-TONE PROBLEM
}

\author{
Christopher S. Withers ${ }^{1}$ And SARAlees NADARAJah ${ }^{2}$
}

\begin{abstract}
We give a stochastic expansion for estimates $\widehat{\theta}$ that minimise the arithmetic mean of (typically independent) random functions of a known parameter $\theta$. Examples include least squares estimates, maximum likelihood estimates and more generally $M$-estimates. This is used to obtain leading cumulant coefficients of $\widehat{\theta}$ needed for the Edgeworth expansions for the distribution and density of $n^{1 / 2}\left(\widehat{\theta}-\theta^{0}\right)$ to magnitude $n^{-3 / 2}$ (or to $n^{-2}$ for the symmetric case), where $\theta^{0}$ is the true parameter value and $n$ is typically the sample size. Applications are given to least squares estimates for both real and complex models. An alternative approach is given when the linear parameters of the model are nuisance parameters. The methods are illustrated with the problem of estimating the frequencies when the signal consists of the sum of sinusoids of unknown amplitudes.
\end{abstract}

Mathematics Subject Classification. 62E17, 62E20.

Received November 13, 2008. Revised March 9, 2009 and June 4, 2009.

\section{INTRODUCTION AND SUMMARY}

Let $\widehat{\theta}$ denote an estimate of $\theta$ in $\mathcal{R}^{p}$ based on a random sample of size $n$. There is a large amount of work on expansions for $\widehat{\theta}-\theta^{0}$, where $\theta^{0}$ is the true value of $\theta$. However, most of the work to date are for the sample mean and functions of it. For example, Monti [5] obtains an expansion for the sample mean up to the second order by expanding the saddlepoint approximation. Booth et al. [1] give tilted expansions of a sample mean from a distribution on $k$ points. Kakizawa and Taniguchi [4] obtain expansions for $P(\widehat{\theta}<x)$ under the assumption that $\widehat{\theta}$ has a cumulant expansion in powers of $n^{-1}$. Gatto and Ronchetti [3] provide approximations for $P(m(\bar{X})<x)$ up to $1+O\left(n^{-1}\right)$ for $m(\cdot)$ a smooth function. For a comprehensive review of the known work, we refer the readers to [8].

The aim of this paper is to provide expansions for those $\widehat{\theta}$ that minimise the arithmetic mean of random functions of $\theta$. Maximum likelihood estimates (MLEs), least squares estimates (LSEs), and more generally $M$-estimates are examples of $\widehat{\theta}$ which minimise a random mean function

$$
\Lambda=\Lambda(\theta)=n^{-1} \sum_{N=1}^{n} \Lambda_{N}(\theta)
$$

\footnotetext{
Keywords and phrases. Bias, edgeworth, maximum likelihood, $M$-estimates, Skewness.

1 Applied Mathematics Group, Industrial Research Limited, Lower Hutt, New Zealand.

2 School of Mathematics, University of Manchester, Manchester M13 9PL, UK; mbbsssn2@manchester.ac.uk
} 
for $\theta$ in $\mathcal{R}^{p}$. If $E \partial \Lambda / \partial \theta=0$ and $E \partial^{2} \Lambda / \partial \theta \partial \theta^{\prime}>0$, then $\widehat{\theta} \rightarrow p \theta^{0}$ as $n \rightarrow \infty$. (We use $\mathcal{R}$ and $\mathcal{C}$ to denote the real and complex numbers).

The contents of this paper are organized as follows. In Section 2 we give a stochastic expansion for $\widehat{\theta}-\theta^{0}$ of the form

$$
\widehat{\theta}-\theta^{0} \approx \sum_{a=1}^{\infty} \delta^{a}
$$

where $\delta^{a}=O_{p}\left(n^{-a / 2}\right)$. (The $a$ in $\delta^{a}$ is a superscript not a power).

In Section 3 we use this to obtain the leading coefficients in the expansions for the cumulants of $\widehat{\theta}$ :

$$
\kappa\left(\widehat{\theta}_{i_{1}}, \ldots, \widehat{\theta}_{i_{r}}\right) \approx \sum_{j=r-1}^{\infty} k_{j}^{i_{1} \ldots i_{r}} n^{-j}
$$

for $r \geq 1$ with $k_{0}^{i}=\theta_{i}^{0}$. This implies that $Y_{n}=n^{1 / 2}\left(\widehat{\theta}-\theta^{0}\right) \rightarrow \mathcal{N}_{p}(0, V)$ with $V=\left(k_{1}^{i_{1} i_{2}}\right)=A^{-1}$ for $A=E \partial^{2} \Lambda / \partial \theta \partial \theta^{\prime}$. The leading bias and skewness coefficients $k_{0}^{i_{1}}$ and $k_{2}^{i_{1} i_{2} i_{3}}$ give the Edgeworth expansion of the distribution (and its derivatives) of $Y_{n}$ to $O\left(n^{-1}\right)$, while the coefficients $k_{2}^{i_{1} i_{2}}$ and $k_{3}^{i_{1} \ldots i_{4}}$ give the Edgeworth expansion of the distribution of $Y_{n}$ to $O\left(n^{-3 / 2}\right)$ and $P\left(Y_{n} \in S\right)$ to $O\left(n^{-2}\right)$ for $S=-S \subset \mathcal{R}^{p}$.

Section 4 applies these results to the LSEs for the general signal plus noise model

$$
Y_{N}=S_{N}(\theta)+e_{N} \text { in } \mathcal{R} \text { or } \mathcal{C}, 1 \leq N \leq n
$$

with $\Lambda_{N}(\theta)=\left|Y_{N}-S_{N}(\theta)\right|^{2} / 2$, where the residuals $e_{1}, \ldots, e_{n}$ are assumed independent with mean zero. While the complex formulation can also be dealt with by the real formulation, there are some significant simplifications in staying with the complex model. The $M$-estimate with respect to a given convex function $\rho$ on $\mathcal{R}$ or $\mathcal{C}$ for the model (1.2) is $\widehat{\theta}$ for $\Lambda_{N}(\theta)=\rho\left(Y_{N}-\rho(\theta)\right)$. For smooth $\rho$ the leading cumulant coefficients were essentially found by this method in the real case in [7].

Section 5 considers two examples on the signal frequency problem

$$
y_{k}=s(\theta)+n_{k} \text { in } \mathcal{C}^{M}, k=1, \ldots, K,
$$

where $n_{k}$ is complex normal with covariance not depending on $\theta$, and the $m$ th component of $s(\theta)$ is

$$
s_{m}(\theta)=\sum_{r=1}^{R} a_{r} \exp \left(j \phi_{r}+j w_{r}\left(m+m_{0}\right) / M\right) \text { for } m=0,1, \ldots, M-1,
$$

where $j=\sqrt{-1}$. The $p=3 R$ parameters are $\theta^{\prime}=\left(a^{\prime}, \phi^{\prime}, w^{\prime}\right)$. So, (1.3) can be written in the form (1.2) with $n=2 k M$. Changing the covariance constant $m_{0}$ is equivalent to reparameterising $\phi_{r}$ : we shall see that if $R=1$ then taking $m_{0}=-(M-1) / 2$ makes the asymptotic covariance of $\widehat{\theta}$ diagonal.

In Section 6 we give a variation of the method for the case when the linear parameters of the model are nuisance parameters.

Appendix A provides a list of summation notations used throughout the paper. Some technical details required for the two examples in Section 5 are given in Appendices B and C. The proofs of all theorems are given in Appendix D.

For $x$ a complex matrix we shall use $x^{\prime}$ to denote its transpose, $\bar{x}$ its complex conjugate, and $x^{*}$ the transpose of its complex conjugate. 


\section{THE STOCHASTIC EXPANSION}

Suppose $\left\{\Lambda_{N}(\theta)\right\}$ are real random functions of $\theta$ in $\mathcal{R}^{p}$. Here we show that $\widehat{\theta}$ minimising $\Lambda=\Lambda(\theta)=$ $n^{-1} \sum_{N=1}^{n} \Lambda_{N}(\theta)$ in $\mathcal{R}^{p}$ has the stochastic expansion

$$
\delta=\widehat{\theta}-\theta^{0}=\sum_{a=1}^{\infty} \delta^{a}
$$

with $\delta^{a}=O_{p}\left(n^{-a / 2}\right)$. To avoid excessive subscripts we shall fix $1 \leq i_{0}, i_{1}, \ldots \leq p$ and set

$$
\begin{aligned}
\delta_{j}^{a} & =\left(\delta^{a}\right)_{i_{j}}, \partial_{j}=\partial / \partial \theta_{i_{j}}, \delta_{j}=(\delta)_{i_{j}}, \Lambda_{\cdot 12 \ldots}=\partial_{1} \partial_{2} \ldots \Lambda(\theta), \\
A_{12 \ldots} & =E \Lambda_{\cdot 12 \ldots}, \text { and } \Delta_{12 \ldots}=\Lambda_{\cdot 12 \ldots}-A_{12 \ldots}
\end{aligned}
$$

Theorem 2.1 gives the first three $\delta^{a}$ explicitly in terms of these $\Delta$ 's and $A$ 's. By (2.1) this gives $\widehat{\theta}$ explicitly in terms of $\Delta$ 's and $A$ 's to $O_{p}\left(n^{-2}\right)$.

For $\widehat{\theta}$ to be a consistent estimate we need to assume that

$$
\begin{aligned}
A_{1} & =E \Lambda_{\cdot 1}=0, \\
\Delta_{1 \ldots r} & =O_{p}\left(n^{-1 / 2}\right) \text { as } n \rightarrow \infty .
\end{aligned}
$$

Typically the model contains a location parameter, and the constraint (2.4) effectively specifies how it is defined, as well as identifying the other parameters of the model. The constraint (2.5) generally follows by the Central Limit Theorem, if the $\left\{\Lambda_{N}(\theta)\right\}$ are independent or weakly dependent.

Theorem 2.1. Suppose $\widehat{\theta}$ is the estimate as defined above satisfying (2.4) and (2.5). Suppose also that the eigenvalues of the $p \times p$ matrix $A=\left(A_{12}: 1 \leq i_{1}, i_{2} \leq p\right)$ are bounded away from zero as $n \rightarrow \infty$, so $A$ has bounded inverse $A^{-1}=\left(A^{12}\right)$ as $n \rightarrow \infty$. Then (2.1) holds. Furthermore,

$$
\begin{aligned}
\delta_{0}^{1}= & -A^{01} \Delta_{1} \\
\delta_{0}^{2}= & A^{01} \Delta_{12} A^{23} \Delta_{3}-B^{045} \Delta_{4} \Delta_{5}, \\
\delta_{0}^{3}= & -A^{01}\left(\Delta_{12} \delta_{2}^{2}-A_{123} \sum_{23}^{2} A^{24} \Delta_{4} \delta_{3}^{2} / 2+\Delta_{123} A^{24} \Delta_{4} A^{35} \Delta_{5} / 2-C_{1}^{567} \Delta_{5} \Delta_{6} \Delta_{7}\right) \\
= & -A^{01}\left\{\Delta_{12}\left(A^{23} \Delta_{34} A^{45} \Delta_{5}-B^{234} \Delta_{3} \Delta_{4}\right)\right. \\
& -2^{-1} A_{123} \sum_{23}^{2} A^{24} \Delta_{4}\left(A^{35} \Delta_{56} A^{67} \Delta_{7}-B^{356} \Delta_{5} \Delta_{6}\right) \\
& \left.+2^{-1} A^{24} A^{35} \Delta_{123} \Delta_{4} \Delta_{5}-C_{1}^{234} \Delta_{2} \Delta_{3} \Delta_{4}\right\}
\end{aligned}
$$

and so on, where $B^{123}=A^{14} A^{25} A^{36} A_{456} / 2$ and $C_{1}^{567}=A_{1234} A^{25} A^{36} A^{47} / 6$.

Note that $B^{045}, B^{234}, B^{356}$ and $C_{1}^{234}$ in (2.6)-(2.8) follow from the definitions given for $B^{123}$ and $C_{1}^{567}$. For example, $B^{045}=A^{04} A^{45} A^{56} A_{456} / 2, B^{234}=A^{24} A^{35} A^{46} A_{456} / 2$ and $C_{1}^{234}=A_{1234} A^{22} A^{33} A^{44} / 6$. The first three $\delta^{a}$ given will be sufficient to obtain the cumulant coefficients needed.

\section{The leading CUmulant COEFFicients}

In this section we give the cumulant coefficients of (1.1) needed for the distribution of $n^{1 / 2}\left(\widehat{\theta}-\theta^{0}\right)$ to $O\left(n^{-3 / 2}\right)$, namely $k_{.1}^{12}, k_{.1}^{0}, k_{.2}^{123}, k_{.2}^{12}, k_{.3}^{1234}$, where we extend the notation of (D.1), (2.3) by setting $k_{. j}^{1 \ldots . . r}=k_{j}^{i_{1} \ldots i_{r}}$. We 
now assume $\Lambda_{1}(\theta), \ldots, \Lambda_{n}(\theta)$ independent. For $\pi$ a sequence of integers in $\{1, \ldots, p\}$, using the dot notation of (2.2) for partial derivatives,

$$
\Lambda_{\cdot \pi}=n^{-1} \sum_{N=1}^{n} \Lambda_{N \cdot \pi}
$$

So, for $\pi_{1}, \pi_{2}, \ldots$ such sequences, the joint cumulants of the $\Lambda_{\cdot \pi}$ are given by

$$
\kappa\left(\Lambda_{\pi_{1}}, \ldots, \Lambda_{\pi_{r}}\right)=n^{1-r}\left[\pi_{1}, \ldots, \pi_{r}\right]
$$

where

$$
\left[\pi_{1}, \ldots, \pi_{r}\right]=n^{-1} \sum_{N=1}^{n} \kappa\left(\Lambda_{N \cdot \pi_{1}}, \ldots, \Lambda_{N \cdot \pi_{r}}\right) .
$$

For example, $[1 \ldots r]=E \Lambda_{\cdot 1 \ldots r}=A_{1 \ldots r}$. We shall give the leading cumulant coefficients we need in terms of these [.] functions.

Set $\delta_{r s}=1$ for $r=s$ and 0 otherwise, and $a_{1 \ldots r}^{b_{1} \ldots b_{r}}=\kappa\left(\delta_{1}^{b_{1}}, \ldots, \delta_{r}^{b_{r}}\right)$. This has an expansion of the form

$$
a_{1 \ldots r}^{b_{1} \ldots b_{r}}=\sum_{j \geq\left(b_{1}+\ldots+b_{r}\right) / 2} a_{1 \ldots r \cdot j}^{b_{1} \ldots b_{r}} n^{-j} .
$$

Also, the left hand side of (1.1) is equal to $\theta_{i_{1}}^{0} \delta_{r 1}+\kappa\left(\delta_{1}, \ldots, \delta_{r}\right)$. Substituting (2.1) into this gives

$$
k_{\cdot j}^{1 \ldots r}=\theta_{i_{1}}^{0} \delta_{r 1} \delta_{j 0}+\sum_{b_{1}+\ldots+b_{r} \leq 2 j} a_{1 \ldots r . j}^{b_{1} \ldots b_{r}}
$$

where $b_{1}, \ldots, b_{r}$ are positive integers.

Theorem 3.1. The coefficients $k_{.1}^{12}, k_{.1}^{0}, k_{.2}^{123}, k_{.2}^{12}$ and $k_{.3}^{1234}$ of (3.3) can be expressed as

$$
\begin{gathered}
k_{.1}^{12}=a_{12.1}^{11}, \quad k_{.1}^{0}=a_{1.1}^{2}, \quad k_{.2}^{123}=a_{123.2}^{111}+\sum_{112}^{3} a_{123.2}^{112}, \\
k_{.2}^{12}=\sum_{12}^{2} a_{12.2}^{12}+\sum_{13}^{2} a_{12.2}^{13}+a_{12.2}^{22}
\end{gathered}
$$

and

$$
k_{.3}^{1234}=a_{1234 \cdot 3}^{1111}+\sum_{1112}^{4} a_{1234 \cdot 3}^{1112}+\sum_{1113}^{4} a_{1234 \cdot 3}^{1113}+\sum_{1122}^{6} a_{1234 \cdot 3}^{1122},
$$

where

$$
\begin{gathered}
k_{.1}^{12}=a_{12.1}^{11}=A^{13} A^{24}[3,4], \\
k_{.1}^{0}=a_{1.1}^{2}=A^{01} A^{23}[12,3]-B^{045}[4,5],
\end{gathered}
$$




$$
\begin{aligned}
& a_{123 \cdot 2}^{111}=-A^{14} A^{25} A^{36}[4,5,6], \\
& a_{123 \cdot 2}^{112}=A^{14} A^{25}\left\{A^{36} A^{78} \sum_{45}^{2}[4,67][5,8]-B^{367} \sum_{45}^{2}[4,6][5,7]\right\} \\
& a_{12 \cdot 2}^{12}=-A^{13} A^{24} A^{56}[3,45,6]+A^{13} B^{245}[3,4,5], \\
& a_{12 \cdot 2}^{13}=A^{13} A^{24}\left\{A^{56} A^{78} \sum^{3}[3,45][67,8]-B^{567} \sum^{3}[3,45][6,7]\right. \\
& -2^{-1} A_{456} \sum_{56}^{2} A^{57}\left(A^{68} A^{9,10} \sum^{3}[3,7][89,10]-B^{689} \sum^{3}[3,7][8,9]\right) \\
& \left.+2^{-1} A^{57} A^{68} \sum^{3}[3,456][7,8]-C_{4}^{567} \sum^{3}[3,5][6,7]\right\} \text {, } \\
& a_{12 \cdot 2}^{22}=A^{13} A^{45} A^{26} A^{78} \sum_{34,5}^{2}[5,8][34,67]-\sum_{12}^{2} A^{13} A^{45} B^{267} \sum_{6,7}^{2}[5,6][7,34] \\
& +B^{134} B^{267} \sum_{34}^{2}[3,6][4,7] \\
& a_{1234 \cdot 3}^{1111}=A^{15} A^{26} A^{37} A^{48}[5,6,7,8] \\
& a_{1234 \cdot 3}^{1112}=A^{15} A^{26} A^{37}\left\{-A^{48} A^{9,10} \sum^{6}[89,10][5,6,7]+B^{489} \sum^{6}[8,9][5,6,7]\right\}, \\
& a_{1234 \cdot 3}^{1113}=A^{15} A^{26} A^{37} A^{48}\left\{A^{9,10} A^{11,12} \sum^{15}[5,6][7,89][10.11,12]\right. \\
& -B^{9,10,11} \sum^{15}[5,6][7,89][10,11] \\
& -2^{-1} A_{89,10} \sum_{9,10}^{2} A^{9,11}\left(A^{10,12} A^{13,14} \sum^{15}[5,6][7,11][12.13,14]\right. \\
& \left.-B^{10,12,13} \sum^{15}[5,6][7,11][12,13]\right) \\
& +2^{-1} A^{9,11} A^{10,12} \sum^{15}[5,6][7,89.10][11,12] \\
& \left.-C_{8}^{9,10,11} \sum^{15}[5,6][7,9][10,11]\right\}
\end{aligned}
$$


and

$$
\begin{aligned}
a_{1234 \cdot 3}^{1122}= & A^{15} A^{26}\left\{A ^ { 3 7 } A ^ { 8 9 } \left[A^{4,10} A^{11,12} \sum^{15}[5,6][78,9][10.11,12]\right.\right. \\
& \left.-B^{4,10,11} \sum^{15}[5,6][78,9][10,11]\right] \\
& -B^{378}\left[A^{4,10} A^{11,12} \sum^{15}[5,6][7,8][10.11,12]\right. \\
& \left.-B^{4,10,11} \sum^{15}[5,6][7,8][10,11]\right\}
\end{aligned}
$$

\section{Least squares estimates}

Here we apply the previous section to LSEs for both real and complex models. We begin with the real model. Suppose we observe

$$
Y=S(\theta)+e \text { in } \mathcal{R}^{n} \text {, that is } Y_{N}=S_{N}(\theta)+e_{N} \text { in } \mathcal{R} \text { for } 1 \leq N \leq n
$$

with $e_{1}, \ldots, e_{n}$ independent and identically distributed (i.i.d.) with mean zero. Denote their $r$ th cumulant by $\lambda_{r}=\kappa_{r}\left(e_{1}\right)$. The LSE is $\widehat{\theta}$ minimising

$$
\Lambda=n^{-1}|Y-S(\theta)|^{2} / 2=n^{-1} \sum_{N=1}^{n} \Lambda_{N}(\theta),
$$

where $\Lambda_{N}(\theta)=\left|Y_{N}-S_{N}(\theta)\right|^{2} / 2$. So,

$$
\Lambda_{N \cdot 1 \ldots r}=\partial_{1} \ldots \partial_{r} S_{N}^{2} / 2-Y_{N} S_{N \cdot 1 \ldots r}, A_{1 \ldots r}=[1 \ldots r]=n^{-1} \sum_{N=1}^{n}\left\{\partial_{1} \ldots \partial_{r} S_{N}^{2} / 2-S_{N} S_{N \cdot 1 \ldots r}\right\} .
$$

For $\pi_{1}, \pi_{2}, \ldots$ sequences of integers in $1 \ldots p$, set

$$
\left\langle\pi_{1}, \pi_{2}, \ldots\right\rangle=n^{-1} \sum_{N=1}^{n} S_{N \cdot \pi_{1}} S_{N \cdot \pi_{2}} \ldots
$$

Theorem 4.1 uses the previous section to express the cumulant coefficients we need in terms of these \langle\rangle functions. Theorem 4.2 notes what form these take for the special case when $A$ is diagonal, having in mind the example of the next section for the case $R=1$.

Theorem 4.1. For the model given by (4.1), the cumulant coefficients of Theorem 3.1 are

$$
\begin{gathered}
k_{1}^{i_{1} i_{2}}=k_{\cdot 1}^{12}=\lambda_{2} A^{12}, \\
k_{1}^{i_{0}}=k_{\cdot 1}^{0}=2^{-1} \lambda_{2} A^{01} A^{23}\{-\langle 1,23\rangle-\langle 2,13\rangle+\langle 3,12\rangle\}, \\
k_{2}^{i_{1} i_{2} i_{3}}=k_{\cdot 2}^{123}=A^{14} A^{25} A^{36}\left\{\lambda_{3}\langle 4,5,6\rangle+\lambda_{2}^{2} \sum_{456}^{3}\langle 4,56\rangle\right\}-6 \lambda_{2}^{2} B^{123},
\end{gathered}
$$




$$
\begin{aligned}
& a_{12 \cdot 2}^{12}=\lambda_{3} A^{13}\left(A^{24} A^{56}\langle 3,45,6\rangle-B^{245}\langle 3,4,5\rangle\right), \\
& a_{12 \cdot 2}^{13} / \lambda_{2}^{2}=A^{13} A^{24} A^{56}\left(A^{78} \sum_{38}^{2}\langle 3,45\rangle\langle 8,67\rangle+\langle 34,56\rangle\right) \\
& -2^{-1} A^{24}\langle 3,45\rangle A^{56} A_{678}\left(A^{13} A^{78}+2 A^{18} A^{37}\right) \\
& +A^{13} A^{24}\left(-2^{-1}\langle 3,45\rangle B^{5}-\langle 6,45\rangle B_{3}^{56}\right. \\
& +2 A_{346} B^{6}+2^{-1} A_{456} A_{378} A^{57} A^{68}+2^{-1}\langle 3,456\rangle A^{56} \\
& \left.+\langle 6,345\rangle A^{56}-2^{-1} A_{3456} A^{56}\right), \\
& a_{12 \cdot 2}^{22} / \lambda_{2}^{2}=A^{13} A^{26}\left(A^{47}\langle 34,67\rangle+A^{45} A^{78}\langle 34,8\rangle\langle 5,67\rangle\right)-2 \sum_{12}^{2} A^{13} B^{246}\langle 6,34\rangle+2 B_{6}^{13} B_{3}^{26}, \\
& a_{1234 \cdot 3}^{1111}=\lambda_{4} A^{15} A^{26} A^{37} A^{48}\langle 5,6,7,8\rangle, \\
& a_{1234 \cdot 3}^{1112}=\left[\lambda_{2} \lambda_{3}\right]\left\{-A^{15} A^{26} A^{37} A^{48} A^{9,10} \sum_{567}^{3}\langle 5,89\rangle\langle 10,6,7\rangle\right. \\
& -A^{48}\left[A^{26} A^{37} A^{19}\langle 89,6,7\rangle+A^{15} A^{37} A^{29}\langle 89,5,7\rangle+A^{15} A^{26} A^{39}\langle 89,5,6\rangle\right] \\
& \left.+A^{15} A^{26} A^{37} A^{48} A^{9,10} \sum_{567}^{3} A_{58,10}\langle 6,7,9\rangle\right\} \text {, } \\
& a_{1234 \cdot 3}^{1113}=\lambda_{2}^{3} A^{15} A^{26} A^{37} A^{48}\left\{A^{9,10} A^{11,12} \sum^{15}\langle 5,6\rangle\langle 7,89\rangle\langle 12,10.11\rangle\right. \\
& -B^{9,10,11} \sum^{15}\langle 5,6\rangle\langle 7,89\rangle\langle 10,11\rangle \\
& -2^{-1} A_{89,10} \sum_{9,10}^{2} A^{9,11}\left(A^{10,12} A^{13,14} \sum^{15}\langle 5,6\rangle\langle 7,11\rangle\langle 12.13,14\rangle\right. \\
& \left.-B^{10,12,13} \sum^{15}\langle 5,6\rangle\langle 7,11\rangle\langle 12,13\rangle\right)+2^{-1} A^{9,11} A^{10,12} \sum^{15}\langle 5,6\rangle\langle 7,89.10\rangle\langle 11,12\rangle \\
& \left.-C_{8}^{9,10,11} \sum^{15}\langle 5,6\rangle\langle 7,9\rangle\langle 10,11\rangle\right\}
\end{aligned}
$$

and

$$
\begin{aligned}
a_{1234 \cdot 3}^{1122}= & \lambda_{2}^{3} A^{15} A^{26}\left\{A ^ { 3 7 } A ^ { 8 9 } \left[A^{4,10} A^{11,12} \sum^{15}\langle 5,6\rangle\langle 78,9\rangle\langle 10.11,12\rangle\right.\right. \\
& \left.-B^{4,10,11} \sum^{15}\langle 5,6\rangle\langle 78,9\rangle\langle 10,11\rangle\right]-B^{378}\left[A^{4,10} A^{11,12} \sum^{15}\langle 5,6\rangle\langle 7,8\rangle\langle 10.11,12\rangle\right. \\
& \left.\left.-B^{4,10,11} \sum^{15}\langle 5,6\rangle\langle 7,8\rangle\langle 10,11\rangle\right]\right\}
\end{aligned}
$$

where $B^{1}=B^{123} A_{23}=2^{-1} A^{12} A_{234} A^{34}$ and $B_{1}^{23}=B^{234} A_{14}=2^{-1} A_{145} A^{24} A^{35}$. 
Theorem 4.2. The corresponding expressions of Theorem 4.1 for $A$ diagonal are

$$
\begin{aligned}
& k_{1}^{i_{0}}=k_{\cdot 1}^{0}=-2^{-1} \lambda_{2} A^{00} A^{22}\langle 0,22\rangle, \\
& k_{2}^{i_{1} i_{2} i_{3}}=k_{\cdot 2}^{123}=A^{11} A^{22} A^{33}\left\{\lambda_{3}\langle 1,2,3\rangle+\lambda_{2}^{2}\left(\sum_{123}^{3}\langle 1,23\rangle-3 A_{123}\right)\right\} \\
& a_{12 \cdot 2}^{12}=\lambda_{3} A^{11}\left\{A^{22} A^{55}\langle 1,25,5\rangle-B^{245}\langle 1,4,5\rangle\right\}, \\
& a_{12 \cdot 2}^{13} / \lambda_{2}^{2}=A^{11} A^{22} A^{33}\left[A^{77}(\langle 1,25\rangle\langle 7,57\rangle+\langle 1,57\rangle\langle 7,25\rangle)+\langle 12,55\rangle\right] \\
& -2^{-1} A^{11} A^{22} A^{55}\left(\langle 1,25\rangle A_{577} A^{77}\right. \\
& \left.+2\langle 3,25\rangle A_{531} A^{33}\right)+A^{11} A^{22}\left[-2^{-1}\langle 1,25\rangle B^{5}\right. \\
& -\langle 6,25\rangle B_{1}^{56}+2 A_{126} B^{6}+2^{-1} A_{156} A_{256} A^{55} A^{66} \\
& \left.+2^{-1}\langle 1,255\rangle A^{55}+\langle 5,125\rangle A^{55}-2^{-1} A_{1255} A^{55}\right] \text {, } \\
& a_{12 \cdot 2}^{22} / \lambda_{2}^{2}=A^{11} A^{22} A^{44}\left(\langle 14,24\rangle+A^{77}\langle 7,14\rangle\langle 4,27\rangle\right)-\sum_{12}^{2} A^{11} B^{246}\langle 6,14\rangle+2 B_{6}^{13} B_{3}^{26}, \\
& a_{1234 \cdot 3}^{1111}=\lambda_{4} A^{11} A^{22} A^{33} A^{44}\langle 1,2,3,4\rangle, \\
& a_{1234 \cdot 3}^{1112} /\left(\lambda_{2} \lambda_{3}\right)=A^{11} \ldots A^{44}\left\{-A^{55} \sum_{123}^{3}\langle 1,45\rangle\langle 2,3,5\rangle-\sum_{123}^{3}\langle 1,2,34\rangle\right. \\
& \left.+A^{55} \sum_{123}^{3} A_{145}\langle 2,3,5\rangle\right\} \\
& a_{1234 \cdot 3}^{1113} / \lambda_{2}^{3}=A^{11} A^{22} A^{33} A^{44}\left\{A^{99} A^{11,11} \sum^{15}\langle 1,2\rangle\langle 3,49\rangle\langle 11,9.11\rangle\right. \\
& -B^{9,10,11} \sum^{15}\langle 1,2\rangle\langle 3,49\rangle\langle 10,11\rangle \\
& -2^{-1} A_{49,10} \sum_{9,10}^{2} A^{9,9}\left(A^{10,10} A^{13,13} \sum^{15}\langle 1,2\rangle\langle 3,9\rangle\langle 10 \cdot 13,13\rangle\right. \\
& \left.-B^{10,12,13} \sum^{15}\langle 1,2\rangle\langle 3,9\rangle\langle 12,13\rangle\right)+2^{-1} A^{99} A^{10,10} \sum^{15}\langle 1,2\rangle\langle 3,49.10\rangle\langle 9,10\rangle \\
& \left.-C_{4}^{9,10,11} \sum^{15}\langle 1,2\rangle\langle 3,9\rangle\langle 10,11\rangle\right\}
\end{aligned}
$$


and

$$
\begin{aligned}
a_{1234 \cdot 3}^{1122} / \lambda_{2}^{3}= & A^{11} A^{22}\left\{A ^ { 3 3 } A ^ { 8 8 } \left[A^{44} A^{11,11} \sum^{15}\langle 1,2\rangle\langle 38,8\rangle\langle 4.11,11\rangle\right.\right. \\
& \left.-B^{4,10,11} \sum^{15}\langle 1,2\rangle\langle 38,8\rangle\langle 10,11\rangle\right]-B^{378}\left[A^{44} A^{11,11} \sum^{15}\langle 1,2\rangle\langle 7,8\rangle\langle 4.11,11\rangle\right. \\
& \left.\left.-B^{4,10,11} \sum^{15}\langle 1,2\rangle\langle 7,8\rangle\langle 10,11\rangle\right]\right\}
\end{aligned}
$$

where $B^{123}=A^{11} A^{22} A^{33} A_{123} / 2, C_{1}^{234}=A_{1234} A^{22} A^{33} A^{44} / 6$, and

$$
B^{1}=2^{-1} A^{11} A^{22} A_{122}, B_{1}^{23}=2^{-1} A_{123} A^{22} A^{33} .
$$

Note that the implicit summations are over the i's not on the left hand side. For example, in (4.14) the summation is over $i_{2}$, not $i_{0}$.

Note that $B^{5}, B^{6}, B_{3}^{56}, B_{6}^{13}$ and $B_{3}^{26}$ in Theorem 4.1 follow from the definitions given for $B^{1}$ and $B_{1}^{23}$. For example, $B^{5}=B^{523} A_{23}, B^{6}=B^{623} A_{23}, B_{3}^{56}=B^{564} A_{34}$ and $B_{6}^{13}=B^{134} A_{64}$. Similar comments apply to Theorem 4.2.

Now suppose we replace the real model (4.1) by the complex model

$$
Y=S(\theta)+e \text { in } \mathcal{C}^{n} \text {, that is } Y_{N}=S_{N}(\theta)+e_{N} \text { in } \mathcal{C} \text { for } 1 \leq N \leq n
$$

with $e_{N}=e_{N 1}+j e_{N 2}$ for $j=\sqrt{-1}$ and $\left\{e_{N 1}, e_{N 2}\right\}$ independent and identically distributed with mean zero and cumulants $\left\{\lambda_{r}\right\}$. The LSE is again given by (4.2). This can be put in the framework of (4.1) with $n$ replaced by $2 n$, (so that $\Lambda$ and $A_{1 \ldots r}$ are half what they are for the complex version with $e_{N 1}=e_{N}$ of (4.1)), but it is simpler to adapt the preceding as follows:

$$
\begin{aligned}
\Lambda_{N \cdot 1 \ldots r} & =\partial_{1} \ldots \partial_{r} \bar{S}_{N} S_{N} / 2-\bar{Y}_{N} S_{N \cdot 1 \ldots r} / 2-Y_{N} \bar{S}_{N \cdot 1 \ldots r} / 2, \\
A_{1 \ldots r} & =[1 \ldots r]=n^{-1} \sum_{N=1}^{n}\left\{\partial_{1} \ldots \partial_{r}\left|S_{N}\right|^{2} / 2-\operatorname{Re} \bar{S}_{N} S_{N \cdot 1 \ldots r}\right\} \\
& =n^{-1}\left(\partial_{1} \ldots \partial_{r}|S|^{2} / 2-\operatorname{Re}^{*} S^{*} S_{1 \ldots r}\right) .
\end{aligned}
$$

(Recall that $\bar{Y}_{N}$ is the complex conjugate of $Y_{N}$, and $S^{*}=\bar{S}^{\prime}$.) Let us extend the notation of (4.3) by writing

$$
\left\langle\bar{\pi}_{1}, \pi_{2}, \pi_{3}\right\rangle=n^{-1} \sum_{N=1}^{n} \bar{S}_{N \cdot \pi_{1}} S_{N \cdot \pi_{2}} S_{N \cdot \pi_{3}}
$$

and so on for $\pi_{1}, \pi_{2}, \ldots$ sequences of integers in $1 \ldots p$. One obtains

$$
\begin{aligned}
n \Lambda_{\cdot 1} & =-\operatorname{Re} e^{*} S_{\cdot 1}, n \Lambda_{\cdot 12}=\operatorname{Re}\left(S_{\cdot 1}^{*} S_{\cdot 2}-e^{*} S_{\cdot 12}\right), \\
A_{12} & =\operatorname{Re} B_{12}=\left(B_{12}+\bar{B}_{12}\right) / 2 \text { for } B_{12}=S_{\cdot 1}^{*} S_{\cdot 2} / n=\langle\overline{1}, 2\rangle, \\
\Lambda_{1 . \ldots r} & =\operatorname{Re}\left(B_{1 \ldots r}-e^{*} S_{1 \ldots .} / n\right), A_{1 \ldots r}=\operatorname{Re} B_{1 \ldots r},
\end{aligned}
$$


where

$$
\begin{aligned}
B_{123} & =\left(\sum_{123}^{3} S_{\cdot 1}^{*} S_{\cdot 23}\right) / n=\sum_{123}^{3}\langle\overline{1}, 23\rangle \\
B_{1234} & =\left(\sum_{1234}^{4} S_{\cdot 1}^{*} S_{\cdot 234}+\sum_{123}^{3} S_{\cdot 12}^{*} S_{\cdot 34}\right) / n=\sum_{1234}^{4}\langle\overline{1}, 234\rangle+\sum_{123}^{3}\langle\overline{12}, 34\rangle .
\end{aligned}
$$

Note that $B_{1 \ldots r}$ can be written down using the form for the partial Bell polynomial $B_{r 2}$ on page 307 of [2]; his $B_{22}=x_{1}^{2}$, his $B_{32}=3 x_{1} x_{2}$, his $B_{42}=4 x_{1} x_{3}+3 x_{2}^{2}$, his $B_{52}=5 x_{1} x_{4}+10 x_{2} x_{3}$ so that

$$
B_{12345}=\left(\sum^{5} S_{\cdot 1}^{*} S_{\cdot 2345}+\sum^{10} S_{\cdot 12}^{*} S_{\cdot 345}\right) / n=\sum^{5}\langle\overline{1}, 2345\rangle+\sum^{10}\langle\overline{12}, 345\rangle,
$$

and so on. We now give the complex form of (D.5). Set $\gamma_{i}=S_{\cdot \pi_{i}}$ and $T_{i}=e^{*} \gamma_{i}$. If $r>1$ then

$$
\begin{aligned}
(-2)^{r}\left[\pi_{1}, \ldots, \pi_{r}\right] & =2^{r} n^{-1} \kappa\left(\operatorname{Re} T_{1}, \ldots, \operatorname{Re} T_{r}\right)=n^{-1} \kappa\left(T_{1}+\bar{T}_{1}, \ldots, T_{r}+\bar{T}_{r}\right) \\
& =\sum_{i=0}^{r} k\left(1^{i} \overline{1}^{r-i}\right) \sum^{\left(\begin{array}{l}
r \\
i
\end{array}\right)}\left\langle\bar{\pi}_{1}, \ldots, \bar{\pi}_{i}, \pi_{i+1}, \ldots, \pi_{r}\right\rangle,
\end{aligned}
$$

where $k\left(1^{i} \overline{1}^{j}\right)=\kappa\left(e_{1}, \cdots, e_{1}, \bar{e}_{1}, \cdots, \bar{e}_{1}\right)$, counting $e_{1} i$ times and $\bar{e}_{1} j$ times. Also the inner summation is over all such $\left(\bar{\pi}_{1}, \ldots, \bar{\pi}_{i}\right)$ giving different terms. These joint cumulants can be written in terms of the real cumulants $\left\{\lambda_{r}\right\}: k\left(1^{i} \overline{1}^{r-i}\right)=\left[1+(-1)^{r-i} j^{r}\right] \lambda_{r}$ so that $k\left(1^{2}\right)=0, k(1 \overline{1})=2 \lambda_{2}$. For example,

$$
\begin{aligned}
4\left[\pi_{1}, \pi_{2}\right] & =E\left(T_{1} T_{2}+\sum^{2} T_{1} \bar{T}_{2}+\bar{T}_{1} \bar{T}_{2}\right) / n=2 \lambda_{2} \sum^{2}\left\langle\bar{\pi}_{1}, \pi_{2}\right\rangle=4 \lambda_{2} \operatorname{Real}\left(\left\langle\bar{\pi}_{1}, \pi_{2}\right\rangle\right), \\
-8\left[\pi_{1}, \pi_{2}, \pi_{3}\right] & =2 \lambda_{3} \operatorname{Real}\left((1+j)\left\{\left\langle\pi_{1}, \pi_{2}, \pi_{3}\right\rangle+\sum^{3}\left\langle\pi_{1}, \bar{\pi}_{2}, \bar{\pi}_{3}\right\rangle\right\}\right), \\
16\left[\pi_{1}, \pi_{2}, \pi_{3}, \pi_{4}\right] & =4 \lambda_{4} \operatorname{Real}\left(\left\{\left\langle\pi_{1}, \pi_{2}, \pi_{3}, \pi_{4}\right\rangle+\sum_{234}^{3}\left\langle\bar{\pi}_{1}, \bar{\pi}_{2}, \pi_{3}, \pi_{4}\right\rangle\right\}\right) .
\end{aligned}
$$

So, by (D.5) the cumulant coefficients for the complex case are obtained from those for the real case by replacing

$$
\begin{aligned}
& \left\langle\pi_{1}, \pi_{2}\right\rangle \text { by } \operatorname{Real}\left(\left\langle\bar{\pi}_{1}, \pi_{2}\right\rangle\right) \text {, } \\
& \left\langle\pi_{1}, \pi_{2}, \pi_{3}\right\rangle \quad \text { by } \quad \text { Real }\left((1+j)\left\{\left\langle\pi_{1}, \pi_{2}, \pi_{3}\right\rangle+\sum^{3}\left\langle\pi_{1}, \bar{\pi}_{2}, \bar{\pi}_{3}\right\rangle\right\}\right) / 4 \text {, } \\
& \left\langle\pi_{1}, \pi_{2}, \pi_{3}, \pi_{4}\right\rangle \text { by } \quad \text { Real }\left(\left\{\left\langle\pi_{1}, \pi_{2}, \pi_{3}, \pi_{4}\right\rangle+\sum_{234}^{3}\left\langle\bar{\pi}_{1}, \bar{\pi}_{2}, \pi_{3}, \pi_{4}\right\rangle\right\}\right) / 4 \text {, }
\end{aligned}
$$


and so on. A simpler way of seeing this - without having to involve the joint cumulants $k\left(1^{i} \overline{1}^{r-i}\right)$, is as follows. Consider the complex numbers $a=a_{1}+j a_{2}, b=b_{1}+j b_{2}, \ldots$ Then $a_{1}=(a+\bar{a}) / 2$ and $a_{2}=-(a-\bar{a}) j / 2$. So,

$$
\begin{aligned}
a_{1} b_{1}+a_{2} b_{2} & =\operatorname{Real}(\bar{a} b), \\
a_{1} b_{1} c_{1}+a_{2} b_{2} c_{2} & =\operatorname{Real}\left((1+j)\left(a b c+\sum^{3} \overline{a b} c\right) / 4,\right. \\
a_{1} b_{1} c_{1} d_{1}+a_{2} b_{2} c_{2} d_{2} & =\operatorname{Real}\left(a b c d+\sum_{b c d}^{3} a b \overline{c d}\right) / 4 \\
& =\operatorname{Real}(a b c d+a b \overline{c d}+a c \overline{b d}+a d \overline{b c}) / 4,
\end{aligned}
$$

and so on. Now take $a=S_{N . \pi_{1}}, b=S_{N . \pi_{2}}, \ldots$ Then $n^{-1} \sum_{N=1}^{n}\left(a_{1} b_{1} c_{1}+a_{2} b_{2} c_{2}\right)$ is twice the real version of $\left\langle\pi_{1}\right.$, $\left.\pi_{2}, \pi_{3}\right\rangle$ for the real version of the complex version with $n$ replaced by $2 n$. By (4.28) it equals the right hand side of (4.27). Similarly, one can write down $\left\langle\pi_{1}, \ldots, \pi_{r}\right\rangle$ for the real version of the complex model in terms of $\left\langle\pi_{1}, \ldots, \pi_{r}\right\rangle$ for the complex model. So, the real versions (4.4), (4.5), (4.6), imply the complex versions

$$
\begin{aligned}
k_{1}^{i_{1} i_{2}} & =k_{\cdot 1}^{12}=\lambda_{2} A^{12}, k_{1}^{i_{0}}=k_{\cdot 1}^{0}=2^{-1} \lambda_{2} A^{01} A^{23} \operatorname{Real}(\{-\langle\overline{1}, 23\rangle-\langle\overline{2}, 13\rangle+\langle\overline{3}, 12\rangle\}), \\
k_{2}^{i_{1} i_{2} i_{3}} & =k_{\cdot 2}^{123}=A^{14} A^{25} A^{36} \operatorname{Real}\left(\lambda_{3}(1+j)\left\{\langle 4,5,6\rangle+\sum\langle 4, \overline{5}, \overline{6}\rangle\right\} / 4+\lambda_{2}^{2} \sum_{456}^{3}\langle\overline{4}, 56\rangle\right)-6 \lambda_{2}^{2} B^{123} .
\end{aligned}
$$

Similarly, $k_{2}^{i_{1} i_{2}}=k_{\cdot 2}^{12}$ can be written down from its real form given by (3.5), (3.11), (3.12), (3.13), and $k_{3}^{i_{1} i_{2} i_{3} i_{4}}=$ $k_{.3}^{1234}$ can be written down from its real form given by (3.6), (3.14)-(3.17). Note that if $e_{1}$ is complex normal with components having the same variance, then (4.26) implies that $\left[\pi_{1}, \ldots, \pi_{r}\right]=0$ for $r>2$ just as this holds by (D.5) for the real case (4.1) if $e_{1} \sim \mathcal{N}\left(0, \lambda_{2}\right)$.

\section{EXAMPLes}

We now drop the convention of Sections 2-4 of suppressing the $i$ 's to the usual convention that

$$
S_{N \cdot i_{1} \ldots i_{r}}=\partial_{i_{1}} \ldots \partial_{i_{r}} S_{N}
$$

for $\partial_{i}=\partial / \partial \theta_{i}$. That is, we write $\left\langle i_{1} \ldots i_{r}\right\rangle$, where we had $\langle 1 \ldots r\rangle, A_{i_{1} i_{2}}=\left\langle i_{1}, i_{2}\right\rangle$, where we had $A_{12}=\langle 1,2\rangle$ in (D.3), $\left\langle i_{1}, i_{2} i_{3}\right\rangle$, where we had $\langle 1,23\rangle$ in (D.4), and so on. So, now

$$
A_{r s}=\langle r, s\rangle=n^{-1} \sum_{N=1}^{n} S_{N \cdot r} S_{N \cdot s}
$$

Example 5.1. Consider the $R$ signal $M$ frequency problem: observe

$$
y_{k}=s(\theta)+n_{k} \text { in } \mathcal{C}^{M} \text { for } k=1, \ldots, K,
$$

where $n_{1}, \ldots, n_{K}$ are independent $\mathcal{C N}_{M}\left(0, v I_{M}\right)$, that is, with real and imaginary parts independent $\mathcal{N}_{M}\left(0, v I_{M} / 2\right)$. (So, $\lambda_{2}$ of Sect. 4 is $v / 2$ and $\lambda_{r}=0$ for $r \neq 2$.) Counting $m=0,1, \ldots, M-1$, suppose that the $m$ th component of $s(\theta)$ has the form

$$
s_{m}(\theta)=\sum_{r=1}^{R} a_{r} \exp \left(j \alpha_{m r}\right)=s_{m 1}+j s_{m 2}
$$


say, for $j=\sqrt{-1}$, where $\alpha_{m r}=\varphi_{r}+\nu_{m} w_{r}$ and $\nu_{m}=\left(m+m_{0}\right) / M$, and $\left\{a_{r}, \varphi_{r}, w_{r}\right\}$ are real so that $p=3 R$ and we can take $\theta^{\prime}=\left(a^{\prime}, \varphi^{\prime}, w^{\prime}\right)$. The main parameter is $w ;(a, \varphi)$ are nuisance parameters. We shall obtain the leading cumulant coefficients firstly by using the real model (4.1), and then for comparison using the complex model (4.24). For the real model the MLE $\widehat{\theta}$ minimises

$$
\begin{gathered}
\sum_{k=1}^{K}\left|y_{k}-s(\theta)\right|^{2} / 2=\sum_{N=1}^{n}\left(Y_{N}-S_{N}(\theta)\right)^{2} / 2, \text { where } n=2 k M, \\
\left\{\left(\begin{array}{c}
Y_{1} \\
S_{1}
\end{array}\right), \ldots,\left(\begin{array}{c}
Y_{n} \\
S_{n}
\end{array}\right)\right\}=\left\{\left(\begin{array}{c}
y_{k m 1} \\
s_{m 1}
\end{array}\right),\left(\begin{array}{c}
y_{k m 2} \\
s_{m 2}
\end{array}\right)\right\}: 0 \leq m<M, 1 \leq k \leq K,
\end{gathered}
$$

and $y_{k m}=y_{k m 1}+j y_{k m 2}$. This puts the problem into the real formulation of (4.1) with $e_{N} \sim \mathcal{N}\left(0, \lambda_{2}\right)$. The constant $m_{0}$ is arbitrary, since it reparameterises $\varphi$. Choose $m_{0}=-(M-1) / 2$, so that $\left\{\nu_{m}\right\}$ have arithmetic mean zero. For $1 \leq r, s \leq R, s_{m 1 \cdot r}=\cos \alpha_{m r}, s_{m 1 \cdot r+R}=-a_{r} \sin \alpha_{m r}, s_{m 1 \cdot r+2 R}=-\nu_{m} a_{r} \sin \alpha_{m r}$, $s_{m 2 \cdot r}=\sin \alpha_{m r}, s_{m 2 \cdot r+R}=a_{r} \cos \alpha_{m r}, s_{m 2 \cdot r+2 R}=\nu_{m} a_{r} \cos \alpha_{m r}$ and

$$
A_{r s}=(2 M)^{-1} \sum_{m=0}^{M-1} \sum_{j=1}^{2} s_{m j \cdot r} s_{m j \cdot s} .
$$

Fix $1 \leq r, s \leq R$ and set

$$
\varphi_{r s}=\varphi_{r}-\varphi_{s}, w_{r s}=w_{r}-w_{s}, \delta_{m}=\alpha_{m r}-\alpha_{m s}=\varphi_{r s}+\nu_{m} w_{r s} .
$$

Then the elements of $A=\left\{A_{a b}: 1 \leq a, b \leq 3 R\right\}$ can be identified as follows.

$$
\left\langle a, a^{\prime}\right\rangle:\langle r, s\rangle=(2 M)^{-1} \sum_{m=0}^{M-1} \cos \delta_{m},
$$

So, $\langle r, r\rangle=2^{-1}$ and for $r \neq s$,

$$
\begin{aligned}
\langle r, s\rangle & =2^{-1} \int_{-1 / 2}^{1 / 2} \cos \left(\varphi_{r s}+x w_{r s}\right) \mathrm{d} x+O\left(M^{-1}\right) \\
& =\left(\cos \varphi_{r s}\right) w_{r s}^{-1} \sin \left(w_{r s} / 2\right)+O\left(M^{-1}\right) \text { as } M \rightarrow \infty, \\
\left\langle a, \varphi^{\prime}\right\rangle:\langle r, s+R\rangle & =a_{s}(2 M)^{-1} \sum_{m=0}^{M-1} \sin \delta_{m}=a_{s} 2^{-1} \int_{-1 / 2}^{1 / 2} \sin \left(\varphi_{r s}+x w_{r s}\right) \mathrm{d} x+O\left(M^{-1}\right) \\
& =a_{s}\left(\sin \varphi_{r s}\right) w_{r s}^{-1} \sin \left(w_{r s} / 2\right)+O\left(M^{-1}\right) \text { if } r \neq s \\
& =0 \text { if } r=s,
\end{aligned}
$$




$$
\begin{aligned}
\left\langle a, w^{\prime}\right\rangle:\langle r, s+2 R\rangle= & a_{s}(2 M)^{-1} \sum_{m=0}^{M-1} \nu_{m} \sin \delta_{m} \\
= & a_{s} 2^{-1} \int_{-1 / 2}^{1 / 2} x \sin \left(\varphi_{r s}+x w_{r s}\right) \mathrm{d} x+O\left(M^{-1}\right) \\
= & a_{s} 2^{-1} \cos \varphi_{r s}\left\{-w_{r s}^{-1} \cos \left(w_{r s} / 2\right)+2 w_{r s}^{-2} \sin \left(w_{r s} / 2\right)\right\} \\
& +O\left(M^{-1}\right) \text { if } r \neq s \\
= & 0 \text { if } r=s, \\
\left\langle\varphi, \varphi^{\prime}\right\rangle:\langle r+R, s+ & R\rangle=a_{r} a_{s}(2 M)^{-1} \sum_{m=0}^{M-1} \cos \delta_{m}=a_{r} a_{s}\langle r, s\rangle, \\
\left\langle\varphi, w^{\prime}\right\rangle:\langle r+R, s+2 R\rangle= & (2 M)^{-1} a_{r} a_{s} \sum_{m=0}^{M-1} \nu_{m} \cos \delta_{m} \\
= & 2^{-1} a_{r} a_{s} \int_{-1 / 2}^{1 / 2} x \cos \left(\varphi_{r s}+x w_{r s}\right) \mathrm{d} x+O\left(M^{-1}\right) \\
= & a_{r} a_{s} \sin \varphi_{r s}\left\{2^{-1} w_{r s}^{-1} \cos \left(w_{r s} / 2\right)-w_{r s}^{-2} \sin \left(w_{r s} / 2\right)\right\} \\
& +O\left(M^{-1}\right) \text { if } r \neq s \\
= & 0 \text { if } r=s,
\end{aligned}
$$

and

$$
\begin{aligned}
\left\langle w, w^{\prime}\right\rangle:\langle r+2 R, s+2 R\rangle= & a_{r} a_{s}(2 M)^{-1} \sum_{m=0}^{M-1} \nu_{m}^{2} \cos \delta_{m} \\
= & a_{r} a_{s} 2^{-1} \int_{-1 / 2}^{1 / 2} x^{2} \cos \left(\varphi_{r s}+x w_{r s}\right) \mathrm{d} x+O\left(M^{-1}\right) \\
= & a_{r} a_{s} \cos \varphi_{r s}\left\{\left(4^{-1} w_{r s}^{-1}-2 w_{r s}^{-3}\right) \sin \left(w_{r s} / 2\right)\right. \\
& \left.+w_{r s}^{-2} \cos \left(w_{r s} / 2\right)\right\}+O\left(M^{-1}\right) \text { if } r \neq s \\
= & a_{r}^{2} v_{2} / 2 \text { if } r=s,
\end{aligned}
$$

where

$$
v_{i}=M^{-1} \sum_{m=0}^{M-1} \nu_{m}^{i}=\int_{-1 / 2}^{1 / 2} x^{i} \mathrm{~d} x+O\left(M^{-1}\right) .
$$

In particular,

$$
\begin{aligned}
& v_{2}=(1-\alpha) / 12, v_{4}=(1-\alpha)(3-7 \alpha) / 240, \\
& v_{6}=(1-\alpha)\left(3-18 \alpha+31 \alpha^{2}\right) / 1344, v_{8}=(1-\alpha)\left(5-55 \alpha+239 \alpha^{2}-381 \alpha^{3}\right) / 11520,
\end{aligned}
$$

and $v_{i}=0$ for $i$ odd, where $\alpha=M^{-2}$. So, $A$ is only diagonal if $R=1$. 
The complex formulation, (4.24) with $n=M k$, is simpler than the real formulation above: choose $\theta$ as above. We have

$$
s_{m \cdot r}=s_{m \cdot r+R} /\left(j a_{r}\right)=s_{m \cdot r+2 R} /\left(j \nu_{m} a_{r}\right)=\exp \left(j \alpha_{m r}\right)
$$

Set

$$
g_{M r}(\delta)=M^{-1} \sum_{m=0}^{M-1} \nu_{m}^{r} \exp \left(j \nu_{m} \delta\right)
$$

A closed form for $g_{M r}$ is given in Appendix C. For example, $g_{M 0}(0)=1$ and if $\delta \neq 0$, then

$$
\begin{aligned}
M g_{M 0}(\delta) & =\{\cos (a-b)-\cos (a+b)\} /(1-\cos 2 b)=\sin a / \sin b \\
g_{0}(\delta) & =\sin a / a \text { for } a=\delta / 2, b=\delta /(2 M) .
\end{aligned}
$$

Note that

$$
g_{M r}(\delta)=g_{r}(\delta)+O\left(M^{-1}\right)
$$

where

$$
g_{r}(\delta)=\int_{-1 / 2}^{1 / 2} x^{r} \exp (j x \delta) \mathrm{d} x=2(-1)^{r} r ! \delta^{-r-1} \sum_{k=0}^{r}(1 /(r-k) !)(\delta / 2)^{r-k} b_{k}
$$

and $b_{k}=\sin ((k \pi+\delta) / 2)$ or $j \cos ((k \pi+\delta) / 2)$ for $r$ even or odd. Since $\left\{-\nu_{m}\right\}=\left\{\nu_{m}\right\}$,

$$
\bar{g}_{M r}(\delta)=g_{M r}(-\delta)=(-1)^{r} g_{M r}(\delta)
$$

so $g_{M r}$ is real for $r$ even, and imaginary for $r$ odd, that is, $h_{M r}(\delta)=j^{-1} g_{M r}(\delta)$ is real for $r$ odd. The $A_{i_{1} \cdots i_{r}}$ can be conveniently written in terms of the real and imaginary parts of the function

$$
G_{r}\left(d_{1}, d_{2}\right)=H_{r}\left(d_{1}, d_{2}\right)+j I_{r}\left(d_{1}, d_{2}\right)=\exp \left(j d_{1}\right) g_{M r}\left(d_{2}\right)=M^{-1} \sum_{m=0}^{M-1} \nu_{m}^{r} \exp \left(j\left(d_{1}+\nu_{m} d_{2}\right)\right) .
$$

So, for $r$ even,

$$
H_{r}\left(d_{1}, d_{2}\right)=\cos d_{1} g_{M r}\left(d_{2}\right), I_{r}\left(d_{1}, d_{2}\right)=\sin d_{1} g_{M r}\left(d_{2}\right)
$$

and for $r$ odd,

$$
H_{r}\left(d_{1}, d_{2}\right)=-\sin d_{1} h_{M r}\left(d_{2}\right), I_{r}\left(d_{1}, d_{2}\right)=\cos d_{1} h_{M r}\left(d_{2}\right)
$$

For example, $B$ of (4.25) is given by $B_{a b}=s_{. a}^{*} s . b / M$. As before fix $1 \leq r, s \leq R$. Then

$$
\begin{aligned}
B_{r s} & =B_{r, s+R} /\left(j a_{s}\right)=B_{r+R, s+R} /\left(a_{r} a_{s}\right)=\exp \left(-j \varphi_{r s}\right) g_{M 0}\left(w_{r s}\right), \\
B_{r, s+2 R} /\left(j a_{s}\right) & =B_{r+R, s+2 R} /\left(a_{r} a_{s}\right)=\exp \left(-j \varphi_{r s}\right) g_{M 1}\left(-w_{r s}\right), \\
B_{r+2 R, s+2 R} & =a_{r} a_{s} \exp \left(-j \varphi_{r s}\right) g_{M 2}\left(w_{r s}\right) .
\end{aligned}
$$


So, $A=A^{\prime}$ is given in terms of $\varphi_{r s}, w_{r s}$ of (5.1) by

$$
\begin{aligned}
A_{r s} & =A_{r+R, s+R} /\left(a_{r} a_{s}\right)=\cos \varphi_{r s} g_{M 0}\left(w_{r s}\right), A_{r, s+R}=a_{s} \sin \varphi_{r s} g_{M 0}\left(w_{r s}\right), \\
A_{r, s+2 R} & =\operatorname{Re} j a_{s} \exp \left(-j \varphi_{r s}\right)(-j) h_{M 1}\left(w_{r s}\right)=a_{s} \cos \varphi_{r s} h_{M 1}\left(w_{r s}\right), \\
A_{r+R, s+2 R} & =-a_{r} a_{s} \sin \varphi_{r s} h_{M 1}\left(w_{r s}\right), A_{r+2 R, s+2 R}=a_{r} a_{s} \cos \varphi_{r s} g_{M 2}\left(w_{r s}\right) .
\end{aligned}
$$

Although $A$ for the real and complex formulations look different, $A$ and $A_{1 \cdots r}$ for the complex formulation are exactly twice what they are for the real formulation, as noted in Section 4.

For both the real and complex models $A_{i_{1} \cdots i_{r}}$ requires 6,10 and 16 formulas for $r=2,3$ and 4 ; we gave these six formulas for $r=2$ above; we now give the ten formulas for $r=3$ for the complex case. We use the notation $1 \leq r, s, t \leq R, r_{i}=r+(i-1) R, s_{i}=s+(i-1) R$ and $t_{i}=t+(i-1) R$. So, $B_{r_{1} s_{1} t_{1}}=0$ and $A_{r s t}=A_{r_{1} s_{1} t_{1}}=0$. Similarly, in terms of the Kronecker delta function $\delta_{i j}=1$ for $i=j$ and 0 for $i \neq j$, we have, for example,

$$
\begin{aligned}
B_{r_{2} s_{2} t_{3}} & =\sum_{r s}^{2} \delta_{s t} a_{r} a_{s} j G_{1}\left(\varphi_{s r}, w_{s r}\right)-\delta_{r s} a_{r} a_{t} j G_{1}\left(\varphi_{r t}, w_{r t}\right), \\
B_{r_{2} s_{3} t_{3}} & =\delta_{s t} a_{r} a_{s} j G_{2}\left(\varphi_{s r}, w_{s r}\right)+\sum_{s t}^{2} \delta_{r t} a_{r} a_{s} j G_{2}\left(\varphi_{r s}, w_{r s}\right), \\
B_{r_{3} s_{3} t_{3}} & =\sum_{r s t}^{3} \delta_{s t} a_{r} a_{s} j G_{3}\left(\varphi_{s r}, w_{s r}\right),
\end{aligned}
$$

so that

$$
\begin{aligned}
A_{r_{1} s_{1} t_{2}} & =\delta_{s t} A_{r s}+\delta_{t r} A_{s t} \\
A_{r_{1} s_{2} t_{2}} & =a_{s}\left(\delta_{r t}-\delta_{s t}\right) \cos \varphi_{r s} g_{M 0}\left(w_{r s}\right)+a_{t} \delta_{r s} \cos \varphi_{r t} g_{M 0}\left(w_{r t}\right) \\
A_{r_{2} s_{2} t_{2}} & =\sum_{r s t}^{3} a_{r} a_{s} \delta_{s t} \sin \varphi_{r s} g_{M 0}\left(w_{r s}\right) \\
A_{r_{1} s_{1} t_{3}} & =\cos \varphi_{r s} \sum_{r s}^{2} \delta_{s t} h_{M 1}\left(w_{r s}\right) \\
& =0 \operatorname{if} r=s \\
A_{r_{1} s_{2} t_{3}} & =\delta_{s t} a_{s} \sin \varphi_{r s} h_{M 1}\left(w_{r s}\right)-\sum_{s t}^{2} \delta_{r t} a_{s} \sin \varphi_{r s} h_{M 1}\left(w_{r s}\right) \\
A_{r_{1} s_{3} t_{3}} & =\delta_{s t} a_{s} \cos \varphi_{r s} g_{M 2}\left(w_{r s}\right)+\sum_{s t}^{2} \delta_{r t} a_{s} \cos \varphi_{t s} g_{M 2}\left(w_{t s}\right) \\
A_{r_{2} s_{2} t_{3}} & =-\sum_{r s}^{2} \delta_{s t} a_{r} a_{s} I_{1}\left(\varphi_{s r}, w_{s r}\right)+\delta_{r s} a_{r} a_{t} I_{1}\left(\varphi_{r t}, w_{r t}\right) \\
A_{r_{2} s_{3} t_{3}} & =-\delta_{s t} a_{r} a_{s} I_{2}\left(\varphi_{s r}, w_{s r}\right)-\sum_{s t}^{2} \delta_{r t} a_{r} a_{s} I_{2}\left(\varphi_{r s}, w_{r s}\right) \\
A_{r_{3} s_{3} t_{3}} & =-\sum_{r s t}^{3} \delta_{s t} a_{r} a_{s} I_{3}\left(\varphi_{s r}, w_{s r}\right)
\end{aligned}
$$

We now specialise the above example to the case $R=1$. 
Example 5.2. Consider the one signal case, $R=1$, of the previous example. By the real formulation above, with $a=a_{1}$,

$$
A=(1 / 2) \operatorname{diag}\left(1, a^{2}, a^{2} v_{2}\right), A^{-1}=\operatorname{diag}\left(2,2 a^{-2}, 2 a^{-2} v_{2}^{-1}\right)
$$

By (4.4), $n \operatorname{covar} \widehat{\theta}=\lambda_{2} A^{-1}+O\left(n^{-1}\right)$. By (4.14),

$$
k_{1}^{i_{0}}=-2^{-1} \lambda_{2} A^{i_{0} i_{0}} \sum_{i_{2}=1}^{3} A^{i_{2} i_{2}}\left\langle i_{0}, i_{2} i_{2}\right\rangle .
$$

Also $s_{m j \cdot 11}=0, s_{m 1 \cdot 12}=-\sin \alpha_{m}, s_{m 1 \cdot 13}=-\nu_{m} \sin \alpha_{m}, s_{m 2 \cdot 12}=\cos \alpha_{m}, s_{m 2 \cdot 13}=\nu_{m} \cos \alpha_{m}, s_{m 1 \cdot 22}=$ $-a \cos \alpha_{m}, s_{m 1 \cdot 23}=-\nu_{m} a \cos \alpha_{m}, s_{m 1 \cdot 33}=-\nu_{m}^{2} a \cos \alpha_{m}, s_{m 2 \cdot 22}=-a \sin \alpha_{m}, s_{m 2 \cdot 23}=-\nu_{m} a \sin \alpha_{m}$ and $s_{m 2.33}=\nu_{m}^{2} a \sin \alpha_{m}$, where $\alpha_{m}=\alpha_{m 1}$. So, the non-zero $\langle i, j k\rangle$ are $\langle 2,12\rangle=-\langle 1,22\rangle=a / 2$ and $\langle 3,13\rangle=$ $-\langle 1,33\rangle=a v_{2} / 2$. So, $k_{1}^{1}=2 \lambda_{2} / a$ and $k_{1}^{2}=k_{1}^{3}=0$. So, $\widehat{\varphi}$ and $\widehat{w}$ have low bias but not $\widehat{a}$. The non-zero $A_{i j k}$, $B^{i j k}$ and $k_{2}^{i j k}$ are $A_{122}=a / 2, A_{133}=a v_{2} / 2, B^{122}=2 a^{-3}, B^{133}=2 a^{-3} v_{2}^{-1}$ and $k_{2}^{122}=-8 \lambda_{2}^{2} a^{-3}=v_{2} k_{2}^{133}$. Since $\widehat{\varphi}$ and $\widehat{w}$ both have $k_{1}^{i}=k_{2}^{i i i}=0$, we should check whether their distributions are symmetric.

Next we show that

$$
k_{2}^{33} / \lambda_{2}^{2}=4 a^{-4}\left(5 v_{2}^{-1}+v_{4} / v_{2}^{3}\right) .
$$

By (4.17), with $i_{5}=i, i_{7}=j$,

$$
\begin{aligned}
a_{33 \cdot 2}^{13} / \lambda_{2}^{2}= & \left(A^{33}\right)^{2} A^{i i}\left[A^{j j} c_{1}+\langle 33, i i\rangle\right]-2^{-1}\left(A^{33}\right)^{2} A^{i i} A^{j j} c_{2} \\
& +\left(A^{33}\right)^{2}\left[-2^{-1}\langle 3,3 i\rangle B^{i}-\langle j, 2 i\rangle B_{1}^{i j}+2 A_{33 i} B^{i}+2^{-1} A_{3 i j}^{2} A^{i i} A^{j j}\right. \\
& \left.+2^{-1}\langle 3,3 i i\rangle A^{i i}+\langle i, 33 i\rangle A^{i i}-2^{-1} A_{33 i i} A^{i i}\right]
\end{aligned}
$$

where $c_{1}=\langle 3,3 i\rangle\langle j, j i\rangle+\langle 3, i j\rangle\langle j, 3 i\rangle$ and $c_{2}=\langle 3,3 i\rangle A_{i j j}+2\langle j, 3 i\rangle A_{3 i j}$. So,

$$
\begin{aligned}
a_{33 \cdot 2}^{13} \lambda_{2}^{-2} A_{33}^{2}= & A^{i i}\left[A^{j j}\left\{c_{1}-2^{-1} c_{2}\right\}+\langle 33, i i\rangle\right. \\
& \left.+2^{-1} A_{3 i j}^{2} A^{j j}+2^{-1}\langle 3,3 i i\rangle+\langle i, 33 i\rangle-2^{-1} A_{33 i i}\right] \\
& +2 A_{33 i} B^{i}-2^{-1}\langle 3,3 i\rangle B^{i}-\langle j, 2 i\rangle B_{1}^{i j} \\
= & g_{i}+f_{i j}=\sum_{i=1}^{3} g_{i}+\sum_{i, j=1}^{3} f_{i j}
\end{aligned}
$$

say. Note that

$$
A_{1133}=\langle 11,33\rangle+2\langle 13,13\rangle+2\langle 1,133\rangle+2\langle 3,113\rangle=0+v_{2}-v_{2}+0=0
$$

since

$$
\langle 13,13\rangle=(2 M)^{-1} \sum_{m=0}^{M-1} \nu_{m}^{2}=2^{-1} v_{2}
$$


Note that

$$
\begin{aligned}
B^{1} & =A^{i i} A_{1 i i}=A^{22} A_{122}+A^{33} A_{133}=2 a^{-2} a / 2+2 a^{-2} v_{2}^{-1} a v_{2} / 2=2 a^{-1}, \\
f_{i j} & =A^{i i}\left[A^{j j}\left\{c_{1}-2^{-1} c_{2}\right\}+2^{-1} A_{3 i j}^{2} A^{j j}\right]-\langle j, 2 i\rangle B_{1}^{i j}, \\
g_{i} & =A^{i i}\left[\langle 33, i i\rangle+2^{-1}\langle 3,3 i i\rangle+\langle i, 33 i\rangle-2^{-1} A_{33 i i}\right]+\left(2 A_{33 i}-2^{-1}\langle 3,3 i\rangle\right) B^{i}, \\
g_{1} & =2\left[\langle 1,331\rangle-2^{-1} A_{3311}\right]+\left(2 A_{331}-2^{-1}\langle 3,31\rangle\right) B^{1} \\
& =-v_{2}+a v_{2} \cdot 2 a^{-1}-\left(a v_{2} / 4\right) \cdot 2 a^{-1}=v_{2} / 2, \\
g_{2} & =2 a^{-2}\left[\langle 22,33\rangle+2^{-1}\langle 3,322\rangle+\langle 2,332\rangle-2^{-1} A_{3322}\right],
\end{aligned}
$$

where

$$
\langle 22,33\rangle=(2 M)^{-1} \sum_{m=0}^{M-1} a^{2} \nu_{m}^{2}=2^{-1} a^{2} v_{2} .
$$

Note that $s_{m 1 \cdot 223}=a \nu_{m} \sin \alpha_{m}, s_{m 1 \cdot 233}=a \nu_{m}^{2} \sin \alpha_{m}, s_{m 1 \cdot 333}=a \nu_{m}^{3} \sin \alpha_{m}, s_{m 2 \cdot 223}=-a \nu_{m} \cos \alpha_{m}, s_{m 2 \cdot 233}=$ $-a \nu_{m}^{2} \cos \alpha_{m}$ and $s_{m 1 \cdot 333}=-a \nu_{m}^{3} \cos \alpha_{m}$, so

$$
\langle 3,322\rangle=-(2 M)^{-1} \sum_{m=0}^{M-1}\left(a \nu_{m}\right)^{2}=-2^{-1} a^{2} v_{2}
$$

and

$$
\langle 2,332\rangle=-(2 M)^{-1} \sum_{m=0}^{M-1} a^{2} \nu_{m}^{2}=-2^{-1} a^{2} v_{2} .
$$

Note that

$$
A_{2233}=\langle 22,33\rangle+2\langle 23,23\rangle+2\langle 2,233\rangle+2\langle 3,223\rangle
$$

and

$$
\langle 23,23\rangle=(2 M)^{-1} \sum_{m=0}^{M-1}\left(\nu_{m} a\right)^{2}=2^{-1} a^{2} v_{2},
$$

so $A_{2233}=2^{-1} a^{2} v_{2}(1+2-2-2)=-2^{-1} a^{2} v_{2}$. So, $g_{2}=v_{2}-2^{-1} v_{2}-v_{2}+2^{-1} v_{2}=0$. Note that

$$
\begin{aligned}
g_{3} & =A^{33}\left[\langle 33,33\rangle+3\langle 3,333\rangle / 2-2^{-1} A_{3333}\right]-2^{-1}\langle 3,33\rangle B^{3}, \\
A_{3333} & =3\langle 33,33\rangle+4\langle 3,333\rangle, \\
\langle 33,33\rangle & =(2 M)^{-1} \sum_{m=0}^{M-1} a^{2} \nu_{m}^{4}=2^{-1} a^{2} v_{4},\langle 3,333\rangle=-(2 M)^{-1} \sum_{m=0}^{M-1} a^{2} \nu_{m}^{4}=-2^{-1} a^{2} v_{4},
\end{aligned}
$$

so $g_{3}=-a^{-2} v_{2}^{-1}\left[2^{-1} a^{2} v_{4}-2^{-1} a^{2} v_{4}\right]=0$. Note that

$$
f_{1 j}=A^{11}\left[A^{j j}\left\{c_{1}-2^{-1} c_{2}\right\}+2^{-1} A_{31 j}^{2} A^{j j}\right]-\langle j, 21\rangle B_{1}^{1 j}
$$

at $c_{1}=\langle 3,31\rangle\langle j, j 1\rangle+\langle 3,1 j\rangle\langle j, 31\rangle$ and $c_{2}=\langle 3,31\rangle A_{1 j j}+2\langle j, 31\rangle A_{31 j}$. So, $f_{11}=0$ and $f_{12}=A^{11}\left[A^{22}\{\langle 3,31\rangle\right.$ $\left.\left.\langle 2,21\rangle-2^{-1}\langle 3,31\rangle A_{122}\right\}\right]-\langle 2,21\rangle B_{1}^{12}$. By $(4.23), B_{1}^{12}=2^{-1} A_{112} A^{11} A^{22}=0$, so

$$
f_{12} / 2=2 a^{-2}\left\{\left(a v_{2} / 2\right)(a / 2)-2^{-1}\left(a v_{2} / 2\right)(a / 2)\right\}=v_{2} / 4, f_{13}=A^{11}\left[A^{33} c_{3}+2^{-1} A_{313}^{2} A^{33}\right]-0
$$


at $c_{3}=2\langle 3,13\rangle^{2}-3.2^{-1}\langle 3,13\rangle A_{133}$. So,

$$
f_{13}=A^{11} A^{33}\left[\langle 3,13\rangle\left\{2\langle 3,13\rangle-3 A_{133} / 2\right\}+2^{-1} A_{133}^{2}\right]=v_{2}, f_{2 j}=A^{22}\left[A^{j j} c_{4}+0\right]-\langle j, 22\rangle B_{1}^{2 j}
$$

at $c_{4}=c_{1}-c_{2} / 2$ and $c_{1}=c_{2}=0$. By (4.23), $B_{1}^{21}=0$ as $B_{1}^{23}=A_{14} B^{234}$ is symmetric in 2, 3. So, $f_{2 j}=0$. Note that

$$
f_{3 j}=A^{33}\left[A^{j j} c_{4}+2^{-1} A_{33 j}^{2} A^{j j}\right]-\langle j, 23\rangle B_{1}^{3 j}, c_{1}=0+\langle 3,3 j\rangle\langle j, 33\rangle, c_{2}=0+2\langle j, 33\rangle A_{33 j} .
$$

So, $f_{32}=f_{33}=0$ and $f_{31}=A^{33} A^{11} c_{5}$ at

$$
c_{5}=c_{4}+2^{-1} A_{133}^{2}=\langle 3,31\rangle\langle 1,33\rangle-\langle 1,33\rangle A_{133}+2^{-1} A_{133}^{2}=a^{2} v_{2}^{2} / 8 .
$$

So, $f_{31}=v_{2} / 2$. So,

$$
a_{33 \cdot 2}^{13} \lambda_{2}^{-2} a^{4} v_{2}^{2} / 4=\sum_{i j} f_{i j}+\sum_{i} g_{i}=3 v_{2} / 2
$$

So, $a_{33 \cdot 2}^{13}=6 \lambda_{2}^{2} a^{-4} v_{2}^{-1}$. By $(4.18)$,

$$
a_{33 \cdot 2}^{22} / \lambda_{2}^{2}=\sum_{i} g_{i}+\sum_{i j} f_{i j}
$$

for $g_{i}=\left(A^{33}\right)^{2} A^{i i}\langle 3 i, 3 i\rangle$ and $f_{i j}=\left(A^{33}\right)^{2} A^{i i} A^{j j}\langle j, 3 i\rangle\langle i, 3 j\rangle+f_{i j}^{1}+f_{i j}^{2}$, where $f_{i j}^{1}=-2 A^{33} B^{3 i j}\langle j, 3 i\rangle$ and $f_{i j}^{2}=2 B_{j}^{3 i} B_{i}^{j j}$, giving $\sum g_{i}=4 a^{-4} v_{2}^{-3}\left(v_{4}+2 v_{2}^{2}\right)$ as before. Note that $\sum_{j} f_{1 j}^{1}=-2 A^{33} A^{133}\langle 3,31\rangle$, $f_{2 j}^{1}=-2 A^{33} \cdot 0=0$ and $f_{3 j}^{1}=-2 A^{33} A^{33 j}\langle j, 31\rangle=0$, giving $\sum_{i j} f_{i j}^{1}=-4 a^{-4} v_{2}^{-1}$. Note that

$$
\begin{aligned}
f_{1 j}^{2} & =\left(A^{33}\right)^{2} A^{11} A^{j j}\langle 31, j\rangle\langle 3 j, 1\rangle+2 B_{j}^{31} B_{1}^{3 j}, \\
f_{11}^{2} & =2\left(B_{1}^{13}\right)^{2}=0 \text { as by }(4.23) B_{1}^{13}=2^{-1} A_{113} A^{11} A^{33}=0, \\
f_{12}^{2} & =2 B_{2}^{31} B_{1}^{32}=0 \text { as } B_{1}^{23}=0, \\
f_{13}^{2} & =2 B_{3}^{31} B_{1}^{33}+\left(A^{33}\right)^{3} 2 A^{11}\langle 31,3\rangle\langle 33,1\rangle, \\
B_{3}^{13} & =2^{-1} A_{133} A^{11} A^{33}, B_{1}^{33}=2^{-1} A_{133}\left(A^{33}\right)^{2} .
\end{aligned}
$$

So,

$$
\begin{aligned}
& f_{13}^{2}=2^{-1} A_{133}^{2} A^{11}\left(A^{33}\right)^{3}+2\left(A^{33}\right)^{3}\langle 1,33\rangle\langle 3,31\rangle=0, \\
& f_{22}^{2}=\left(A^{33}\right)^{2}\left(A^{22}\right)^{2}\langle 32,2\rangle^{2}+2\left(B_{2}^{32}\right)^{2}=0
\end{aligned}
$$

as $B_{2}^{32}=2^{-1} A_{223}^{0} A^{33} A^{22}=0$. Note that

$$
f_{23}^{2}=\left(A^{33}\right)^{2} A^{22}\langle 32,3\rangle\langle 33,2\rangle+2 B_{3}^{32} B_{2}^{33}=0, f_{33}^{2}=\left(A^{33}\right)^{4}\langle 3,33\rangle^{2}+2\left(B_{3}^{33}\right)^{2}=0 .
$$

So, $\sum f_{i j}^{2}=0$. So, $a_{33 \cdot 2}^{22} / \lambda_{2}^{2}=4 a^{-4} v_{2}^{-3}\left(v_{4}+2 v_{2}^{2}\right)$. So, (5.2) holds: compare it with $k_{1}^{33} / \lambda_{2}=A^{33}=2 a^{-2} v_{2}^{-1}$. So,

$$
\operatorname{var} \widehat{w}=2 \lambda_{2} a^{-2} v_{2}^{-1} n^{-1}+4 \lambda_{2}^{2} a^{-4}\left(5 v_{2}^{-1}+v_{4} v_{2}^{-3}\right) n^{-2}+O\left(n^{-3}\right)
$$


at $n=2 K M$ and $\lambda_{2}=v / 2$. Set SNR $=a^{2} /(v / K M)$ so that $S N R^{-1}=v /\left(a^{2} K M\right)$. So,

$$
\begin{aligned}
\operatorname{var} \widehat{w} & =2^{-1} v_{2}^{-1} S N R^{-1}\left[1+2^{-1} S N R^{-1}\left(5+v_{4} / v_{2}^{2}\right)\right]+O\left(S N R^{-3}\right) \\
& =6\left(1-M^{-2}\right)^{-1} S N R^{-1}\left[1+S N R^{-1} 3.4\left(1+O\left(M^{-2}\right)\right)\right]+O\left(S N R^{-3}\right)
\end{aligned}
$$

since $v_{2}=12^{-1}\left(1-M^{-2}\right)$ and $v_{4}=80^{-1}+O\left(M^{-2}\right)$.

\section{WHEN LINEAR PARAMETERS ARE NUISANCE PARAMETERS}

Here we give an approach that allows us to reduce the dimension of the parameter space by eliminating nuisance parameters that enter the model linearly, when using the LSE. (For example, in Example 5.1, $\theta$ has dimension $3 R$, but the parameter of interest $w$ has dimension only $R$.)

The method of Section 2 does not require $\Lambda=\Lambda(\theta)$ to be the mean of random functions $\Lambda_{1}(\theta), \ldots, \Lambda_{n}(\theta)$, but only that $A_{1}=0, A=\left(A_{12}\right)$ is bounded away from zero, $A_{1 \ldots r}$ is bounded for $r \geq 1$, and

$$
\Delta_{\cdot 1 \ldots r}=O_{p}\left(n^{-1 / 2}\right) .
$$

First consider again the real model (4.1) $Y_{N}=x_{N}^{\prime}(w) \beta+e_{N}$ in $\mathcal{C}$ with $\beta$ in $\mathcal{R}^{q}, w$ in $\mathcal{R}$, that is $Y=X(w) \beta+e$ in $\mathcal{R}^{n}$, where $X(w)^{\prime}=\left(x_{1} \cdots x_{n}\right)=X$ and $x_{N}=x_{N}(w)$. That is, we now suppose that $\theta=\left(\begin{array}{l}\beta \\ w\end{array}\right)$ and $S_{N}(\theta)=x_{N}(w)^{\prime} \beta$. So, $S(\theta)=S=X(w) \beta$. Set

$$
\Lambda(w)=\inf _{\beta} n^{-1} \sum_{N=1}^{n}\left(Y_{N}-X_{N}^{\prime} \beta\right)^{2}=n^{-1}|Y-X \widehat{\beta}(w)|^{2}=n^{-1} Y^{\prime} Q Y,
$$

where $I-Q=I-Q(w)=P=X\left(X^{\prime} X\right)^{-1} X^{\prime}$ and $\widehat{\beta}(w)=\left(X^{\prime} X\right)^{-1} X^{\prime} Y$, assuming $X^{\prime} X$ has full rank $q \leq n$. So, the LSE is

$$
\widehat{\theta}=\left(\begin{array}{c}
\widehat{\beta}(\widehat{w}) \\
\widehat{w}
\end{array}\right)
$$

where $\widehat{w}$ minimises $\Lambda(w)$. Theorem 6.1 verifies that the conditions (6.1) hold with $(\theta, \Lambda(\theta))$ replaced by $(w, \Lambda(w))$.

Theorem 6.1. Consider the model (4.1). Assume that $X^{\prime} X$ has full rank $q \leq n$ and that $x_{N}$ satisfies

$$
x_{N}(w)=y(N / n, w)=y(N / n)
$$

for some function $y(t, w)$. Also assume that $e_{1}, \ldots, e_{n}$ are i.i.d. in $\mathcal{R}$ with mean zero and cumulants $\left\{\lambda_{r}\right\}$. Finally, assume that

$$
\operatorname{det}(T(1: 2)) \neq 0
$$

where $T\left(\pi_{1}: \pi_{2}\right)=T\left(\pi_{1}, \pi_{2}\right)-T\left(\cdot, \pi_{1}\right)^{\prime} T^{-1} T\left(\cdot, \pi_{2}\right)$, where $T\left(\pi_{1}, \pi_{2}\right)=\int_{0}^{1} y \cdot \pi_{1}(t) y_{\pi_{2}}(t)^{\prime} \mathrm{d} t$, and $\cdot \pi$ denotes differentiation with respect to $w$, not $t$. Then $A_{1}=0, A=\left(A_{12}\right)$ is bounded away from zero, $A_{1 \ldots r}$ is bounded for $r \geq 1$, and (6.1) holds.

We now consider how to adapt Section 3. Since $\Lambda(w)$ is no longer a sum of independent random variables, (3.1) with (3.2) no longer applies. So, let us take (3.1) as the definition of [.]. To be of use we need [.] to be bounded as $n \rightarrow \infty$. Note that $[\pi]=A_{\pi}$ is bounded. For $r>1$,

$$
\left[\pi_{1}, \ldots, \pi_{r}\right]=n^{r-1} \kappa\left[\Delta_{\pi_{1}}, \ldots, \Delta_{\pi_{r}}\right],\left[\pi_{1}, \pi_{2}\right]=n^{-1} \kappa\left[e^{\prime} Q \cdot \pi_{1} S+e^{\prime} Q \cdot \pi_{1} e, e^{\prime} Q \cdot \pi_{2} S+e^{\prime} Q \cdot \pi_{2} e\right] .
$$


Clearly $\left\{\Delta_{\pi_{1}}\right\}$ has $r$ th-order cumulants $O\left(n^{1-r}\right)$ :

$$
\kappa_{r}\left(\Delta_{\pi_{1} 1}, \ldots, \Delta_{\pi_{r} r}\right)=n^{1-r}\left[\pi_{1}, \ldots, \pi_{r}\right]_{1}
$$

where

$$
\begin{aligned}
{\left[\pi_{1}, \ldots, \pi_{r}\right]_{1} } & =\lambda_{r}\left\langle\gamma_{\pi_{1}}, \ldots, \gamma_{\pi_{r}}\right\rangle_{1} \\
\left\langle\gamma_{\pi_{1}}, \ldots, \gamma_{\pi_{r}}\right\rangle_{1} & =n^{-1} \sum_{N=1}^{n} \gamma_{\pi_{1} N} \ldots \gamma_{\pi_{r} N}, \gamma_{\pi}=\left(\gamma_{\pi_{N}}\right)=Q \cdot \pi
\end{aligned}
$$

By (D.12),

$$
n^{2} E \Delta_{\pi_{1} 1} \Delta_{\pi_{2} 2}=\lambda_{4} \sum_{N=1}^{n} Q_{N N \cdot \pi_{1}} Q_{N N \cdot \pi_{2}}+2 \lambda_{2}^{2} \operatorname{tr} Q_{\cdot \pi_{1}} Q_{\cdot \pi_{2}}=O(1)
$$

Similarly, one expects the moments and cumulants of $\left\{n \Delta_{\pi_{2}}\right\}$ to be $O(1)$, and so the $r$ th-order cross-cumulants of $\left\{\Delta_{\pi_{2}}\right\}$ to be $O\left(n^{-r}\right)$. So, one expects the $r$ th-order cross-cumulants of $\left\{\Delta_{\pi_{1} 1}, \Delta_{\pi_{2} 2}\right\}$ to be $O\left(n^{1-r}\right)$ or $O\left(n^{-r}\right)$ and so the [.] functions of (3.2) to be bounded. So, the results of Section 3 should hold with [.] defined by (3.1), $\operatorname{not}(3.2)$.

The complex case is similar: observe

$$
Y_{N}=x_{N}^{*} \beta+e_{N} \text { in } \mathcal{C} \text { with } \beta \text { in } \mathcal{C}^{q}, x_{N}=x_{N}(w),
$$

that is $Y=X \beta+e$ in $\mathcal{C}^{n}$, where $X^{*}=\left(x_{1} \cdots x_{n}\right)$. Assume that $e_{N}=e_{N 1}+j e_{N 2}$ for $j=\sqrt{-1}$ with $\left\{e_{N k}\right\}$ i.i.d. with mean zero and $r$ th cumulants $\lambda_{r} / 2$. So, $\lambda_{2}=E\left|e_{N}\right|^{2}$. Set

$$
\Lambda(w)=\inf _{\beta} n^{-1} \sum_{N=1}^{n}\left|Y_{N}-x_{N}^{*} \beta\right|^{2}=n^{-1}|Y-X \widehat{\beta}(w)|^{2}=n^{-1} Y^{*} Q Y,
$$

where $Q=Q(w)=I-P, P=X\left(X^{*} X\right)^{-1} X^{*}$ and $\widehat{\beta}(w)=\left(X^{*} X\right)^{-1} X^{*} Y$, assuming $X^{*} X$ has full rank $q \leq n$. So, the LSE is

$$
\widehat{\theta}=\left(\begin{array}{c}
\widehat{\beta}(\widehat{w}) \\
\widehat{w}
\end{array}\right)
$$

where $\widehat{w}$ minimises $\Lambda(w)$. Again we assume (6.2) and (6.3) hold with ' replacing * in (D.9)-(D.11) so that $A_{1}=0, A_{1 \ldots r}$ is bounded, $A=\left(A_{12}\right)$ is bounded away from zero. Note that $A_{1 \ldots r}$ is given by (D.6)-(D.9) and their extensions, with' replacing *. Also

$$
\Delta_{\pi}=2 \Delta_{1 \pi}+\Delta_{2 \pi}
$$

for $2 n \Delta_{1 \pi}=2 \operatorname{Re} e^{*} \gamma_{\pi}=e^{*} \gamma_{\pi}+\gamma_{\pi}^{*} e, \gamma_{\pi}=Q_{\cdot \pi} S$ and $n \Delta_{2 \pi}=e^{*} Q_{\cdot \pi} e$, where again $S=S(\theta)=X \beta$. The argument that $\Delta_{\pi}$ is $O_{p}\left(n^{-1 / 2}\right)$ carries over. If $w$ lies in $\mathcal{R}^{q}$ then $A_{\pi}$ and $\Delta_{\pi}$ are still real.

Example 6.1. Observe $y_{k}=S+\varepsilon_{k}$ in $\mathcal{C}^{n}$ for $k=1, \ldots, K$ with $\varepsilon_{k} \sim \mathcal{C N}\left(0, V I_{n}\right)$ for some scalar $V>0$. So, $\left\{y_{k}\right\}$ have arithmetic mean $Y=S+e$ with $e \sim \mathcal{C N}\left(0, \nu I_{n}\right)$ and $\nu=K^{-1} V$. (So, $\lambda_{2}=\nu / 2$ and $\lambda_{r}=0$ for $r \neq 2$.) Suppose $Y=\left(Y_{0}, \ldots, Y_{n-1}\right)^{\prime}$ and $S=\left(S_{0}, \ldots, S_{n-1}\right)^{\prime}$, where $S_{N}=x_{N} \beta$ for $\beta$ in $\mathcal{C}, x_{N}=\exp \left(j \gamma_{N}\right)$, $j=\sqrt{-1}, \gamma_{N}=\nu_{N} w, \nu_{N}=\left(N+N_{0}\right) / n$ and $N_{0}=-(n-1) / 2$. So, $q=1, X^{*} X=n, I-Q=P=n^{-1} X X^{*}$ has $(a, b)$ element

$$
n^{-1} x_{a}^{*} x_{b}=n^{-1} \exp \left\{j\left(\gamma_{b}-\gamma_{a}\right)\right\}=n^{-1} \exp \{j w(b-a) / n\}
$$


and $\Lambda(w)=n^{-1}|Y|^{2}-n^{-2}\left|X^{*} Y\right|^{2}$ and $X^{*} Y=\sum_{N=0}^{n-1} x_{N} Y_{N}$. Since $q=1$ we now replace $1 \ldots r$, where it occurs by $r$ and use the convention $f(w)_{. r}=(\partial / \partial w)^{r} f(w)$ for any smooth function $f(w)$. For example, $x_{N \cdot r}=(\partial / \partial w)^{r} x_{N}=\left(j \nu_{N}\right)^{r} x_{N}$. So,

$$
X_{\cdot r}=\left(x_{0 \cdot r}, \ldots, x_{n-1 \cdot r}\right)^{*}=(-j)^{r}\left(\nu_{0}^{r} x_{0}, \ldots, \nu_{n-1}^{r} x_{n-1}\right)^{*}, X_{\cdot r}^{*} X_{\cdot s}=(-1)^{s} j^{r+s} n v_{r+s}
$$

for $v_{r}=n^{-1} \sum_{N=0}^{n-1} \nu_{N}^{r}$. So,

$$
\begin{aligned}
& v_{1}=0, v_{2}=\left(1-n^{-2}\right) / 12, v_{4}=\left(1-n^{-2}\right)\left(1-7 n^{-2} / 3\right) / 80, \\
& v_{r}=2^{-r} /(r+1)+O\left(n^{-2}\right), \\
& X_{r}^{*} X / n=j^{r} v_{r}, X_{\cdot 1}^{*} X=0, X_{\cdot 1}^{*} X_{\cdot 1} / n=v_{2} \text {, } \\
& Q \cdot 1=-P \cdot 1=-n^{-1}\left(X_{\cdot 1} X^{*}+X X_{\cdot 1}^{*}\right), Q \cdot 2=-n^{-1}\left(X_{\cdot 2} X^{*}+2 X_{\cdot 1} X_{\cdot 1}^{*}+X X_{\cdot 2}^{*}\right), \\
& Q_{\cdot 3}=-n^{-1}\left(X_{.3} X^{*}+3 X_{\cdot 2} X_{\cdot 1}^{*}+3 X_{\cdot 1} X_{\cdot 2}^{*}+X X_{\cdot 3}^{*}\right), \\
& Q_{.4}=-n^{-1}\left(X_{.4} X^{*}+4 X_{.3} X_{.1}^{*}+6 X_{.2} X_{.2}^{*}+4 X_{.1} X_{.3}^{*}+X X_{.4}^{*}\right), \\
& Q .{ }_{1} X=-X_{1}, Q \cdot{ }_{2} X=-\left(X .{ }_{2}-X v_{2}\right), Q .3 X=-\left(X{ }_{.3}-3 X{ }_{.1} v_{2}-X j v_{3}\right), \\
& Q \cdot{ }_{4} X=-\left(X .4-6 X_{\cdot 2} v_{2}-4 X .1 j v_{3}+X v_{4}\right), \\
& X^{*} Q_{\cdot 2} X / n=2 v_{2}, X^{*} Q \cdot 3, X / n=0, X^{*} Q_{\cdot 4} X / n=-2\left(v_{4}+3 v_{2}^{2}\right) .
\end{aligned}
$$

By (D.6), $A_{r}=|\beta|^{2} X^{*} Q_{. r} X / n$, so $A_{2}=2|\beta|^{2} v_{2}, A_{3}=0$ and $A_{4}=-2|\beta|^{2}\left(v_{4}+3 v_{2}^{2}\right)$. Also var $\widehat{w}=k_{21} n^{-1}+$ $k_{22} n^{-2}+\ldots$, where by $(3.5),(3.6)-(3.11)$, replacing $[1,111]$ by $[1,3]$ and so on,

$$
\begin{aligned}
& k_{21}=A_{2}^{-2}[1,1], k_{22}=2 a^{12}+2 a^{13}+a^{22}, \\
& a^{12}=-A_{2}^{-3}[1,1,2], a^{22}=A_{2}^{-4}\left([1,1][2,2]+[1,2]^{2}\right), \\
& a^{13}=A_{2}^{-4}\left(2[1,2]^{2}+[1,1][2,2]+3[1,1][1,3] / 2\right)-A_{2}^{-5} A_{4}[1,1]^{2} / 2,
\end{aligned}
$$

giving

$$
k_{22}=-2 A_{2}^{-3}[1,1,2]+A_{2}^{-4}\left(5[1,2]^{2}+3[1,1][2,2]+3[1,1][1,3]\right)-A_{2}^{-5} A_{4}[1,1]^{2} .
$$

By (6.4), $n \Delta_{r}=e^{*} \gamma_{r}+\gamma_{r}^{*} e+e^{*} Q_{. r} e$ for $\gamma_{r}=Q . r S$. By Appendix B,

$$
\begin{aligned}
E n^{2} \Delta_{r} \Delta_{s} & =E\left(\sum_{r s}^{2} \gamma_{r}^{*} e e^{*} \gamma_{s}+e^{*} Q \cdot r e e^{*} Q \cdot s e\right)=\nu \sum_{r s}^{2} \gamma_{r}^{*} \gamma_{s}+\nu^{2}(\operatorname{tr} Q . r \operatorname{tr} Q . s+\operatorname{tr} Q \cdot r Q \cdot s) \\
& =n \nu|\beta|^{2}\left(d_{r s}+d_{r s}^{*}\right)+\nu^{2} D_{r s},
\end{aligned}
$$

where $d_{r s}=(Q . r X)^{*}\left(Q .{ }_{s} X\right) / n$ and $D_{r s}=\operatorname{tr} Q . r Q . s$ are $O(1)$. In particular, $d_{11}=v_{2}, d_{12}=X_{.1}^{*}\left(X .{ }_{2}-X v_{2}\right) / n=$ $-j v_{3}, d_{13}=-v_{4}-3 v_{2}^{2}, d_{22}=v_{4}+3 v_{2}^{2}, D_{11}=2 v_{2}, D_{12}=0, D_{13}=2\left(v_{4}-3 v_{2}^{2}\right)$ and $D_{22}=2\left(v_{4}+v_{2}^{2}\right)$. So,

$$
\begin{aligned}
& {[1,1]=n E \Delta_{1}^{2}=2 \nu|\beta|^{2} v_{2}(1+\varepsilon) \text { for } \varepsilon=\nu n^{-1}|\beta|^{-2},} \\
& {[1,2]=n E \Delta_{1} \Delta_{2}=0,[1,3]=n E \Delta_{1} \Delta_{3}=-2 \nu|\beta|^{2}\left\{v_{4}+3 v_{2}^{2}-\varepsilon\left(v_{4}-3 v_{2}^{2}\right)\right\},} \\
& {[2,2]=2 \nu|\beta|^{2}\left\{v_{4}+3 v_{2}^{2}+\varepsilon\left(v_{4}+v_{2}^{2}\right)\right\} .}
\end{aligned}
$$

Also for $q_{r}=e^{*} Q . r e$ and $T_{r}=\operatorname{tr} Q . r=0$, by Appendix B

$$
\begin{aligned}
n[1,1,2] & =n^{3} E \Delta_{1}^{2} \Delta_{2}=E\left(2 e^{*} \gamma_{1} \gamma_{1}^{*} e+q_{1}^{2}\right) q_{2} \\
& =2 \nu^{2}\left(\gamma_{1}^{*} \gamma_{1} T_{2}+\gamma_{1}^{*} Q .2 \gamma_{1}\right)+\nu^{3}\left(T_{1}^{2} T_{2}+2 T_{1} \operatorname{tr} Q .{ }_{1} Q .2 T_{2} \operatorname{tr} Q_{\cdot 1}^{2}+2 \operatorname{tr} Q_{\cdot 1}^{2} Q .2\right) \\
& =2 n \nu^{2}|\beta|^{2} d_{121}+2 \nu^{3} D_{112},
\end{aligned}
$$


where $d_{121}=\left(Q \cdot{ }_{1} X\right)^{*} Q_{\cdot 2}\left(Q \cdot{ }_{1} X\right) / n=-2 v_{2}^{2}$ and $D_{112}=\operatorname{tr} Q_{\cdot 1}^{3} Q \cdot 2=0$. So, var $\widehat{w}=2^{-1} v_{2}^{-1} \varepsilon+c \varepsilon^{2}+O\left(\varepsilon^{3}\right)$, where

$$
\begin{aligned}
c & =2^{-1} v_{2}^{-1}\left(\text { from } k_{21}\right)+v_{2}^{-1}\left(\text { from } 2 a^{13}\right)+2^{-2} v_{2}^{-3}\left(v_{4}+3 v_{2}^{2}\right)\left(\text { from } 2 a^{13}+a^{22}\right) \\
& =6 \times 27 / 5+O\left(n^{-2}\right) .
\end{aligned}
$$

So, $\operatorname{var} \widehat{w}=6 \varepsilon(1+5.4 \varepsilon)+O\left(\varepsilon^{3}\right)$.

\section{Appendix A}

The following is the list of distinct summation notations used in the paper:

$$
\begin{aligned}
& \sum_{i j}^{2} f_{i j}=f_{i j}+f_{j i}, \sum_{i j k}^{3} f_{i j k}=f_{i j k}+f_{k i j}+f_{j k i}, \sum^{3} f_{i j} g_{k l}=f_{i j} g_{k l}+f_{i k} g_{j l}+f_{i l} g_{j k}, \\
& \sum_{i j k l}^{4} f_{i j k l}=f_{i j k l}+f_{l i j k}+f_{k l i j}+f_{j k l i}, \sum_{i j k l m}^{5} f_{i j k l m}=f_{i j k l m}+f_{m i j k l}+f_{l m i j k}+f_{k l m i j}+f_{j k l m i}, \\
& \sum_{i j k}^{6} f_{i j k}=f_{i j k}+f_{i k j}+f_{j i k}+f_{j k i}+f_{k i j}+f_{k j i} \\
& \sum_{i i j j}^{6} f_{i i j j}=f_{i i j j}+f_{i j i j}+f_{i j j i}+f_{j i i j}+f_{j i j i}+f_{j j i i}, \\
& \sum^{6} f_{i j} g_{k l}=f_{i j} g_{k l}+f_{i k} g_{j l}+f_{i l} g_{j k}+f_{j k} g_{i l}+f_{j l} g_{i k}+f_{k l} g_{i j} \\
& \sum^{6} g_{l m} f_{i j k}=g_{i l} f_{j k m}+g_{i m} f_{j k l}+g_{j l} f_{i k m}+g_{j m} f_{i k l}+g_{k l} f_{i j m}+g_{k m} f_{i j l}, \\
& \sum^{10} f_{i j} g_{k l m}=f_{i j} g_{k l m}+f_{i k} g_{j l m}+f_{i l} g_{j k m}+f_{i m} g_{j k l}+f_{j k} g_{i l m}+f_{j l} g_{i k m}+f_{j m} g_{i k l}+f_{k l} g_{i j m} \\
& +f_{k m} g_{i j l}+f_{l m} g_{i j k}, \\
& \sum^{10} f_{i j k} g_{l m n}=f_{i j k} g_{l m n}+f_{i j l} g_{k m n}+f_{i j m} g_{k l n}+f_{i j n} g_{k l m}+f_{i k l} g_{j m n}+f_{i k m} g_{j l n}+f_{i k n} g_{j l m} \\
& +f_{i l m} g_{j k n}+f_{i l n} g_{j k m}+f_{i m n} g_{j k l}, \\
& \sum^{12} f_{i l} g_{j k m n}=f_{i l} g_{j k m n}+f_{i m} g_{j k l n}+f_{i n} g_{j k l m}+f_{j l} g_{i k m n}+f_{j m} g_{i k l n}+f_{j n} g_{i k l m}+f_{k l} g_{i j m n} \\
& +f_{k m} g_{i j l n}+f_{k n} g_{i j l m}+f_{l m} g_{i j k n}+f_{l n} g_{i j k m}+f_{m n} g_{i j k l}, \\
& \sum^{15} f_{i j} g_{k l} h_{m n}=f_{i j} g_{k l} h_{m n}+f_{i k} g_{j l} h_{m n}+f_{i l} g_{j k} h_{m n}+f_{i m} g_{j k} h_{l n}+f_{i n} g_{j k} h_{l m}+f_{j k} g_{i l} h_{m n} \\
& +f_{j l} g_{i k} h_{m n}+f_{j m} g_{i k} h_{l n}+f_{j n} g_{i k} h_{l m}+f_{k l} g_{i j} h_{m n}+f_{k m} g_{i j} h_{l n}+f_{k n} g_{i j} h_{l m} \\
& +f_{l m} g_{i j} h_{k n}+f_{l n} g_{i j} h_{k m}+f_{m n} g_{i j} h_{k l},
\end{aligned}
$$




$$
\begin{aligned}
& \sum_{\pi_{1}, \pi_{3}}^{2}\left[\pi_{1}, \pi_{2}\right]\left[\pi_{3}, \pi_{4}\right]=\left[\pi_{1}, \pi_{2}\right]\left[\pi_{3}, \pi_{4}\right]+\left[\pi_{3}, \pi_{2}\right]\left[\pi_{1}, \pi_{4}\right] \\
& \sum_{\pi_{2}, \pi_{3}}^{2}\left[\pi_{1}, \pi_{2}\right]\left[\pi_{3}, \pi_{4}\right]=\left[\pi_{1}, \pi_{2}\right]\left[\pi_{3}, \pi_{4}\right]+\left[\pi_{1}, \pi_{3}\right]\left[\pi_{2}, \pi_{4}\right] \\
& \sum^{3}\left[\pi_{1}, \pi_{2}\right]\left[\pi_{3}, \pi_{4}\right]=\left[\pi_{1}, \pi_{2}\right]\left[\pi_{3}, \pi_{4}\right]+\left[\pi_{1}, \pi_{3}\right]\left[\pi_{2}, \pi_{4}\right]+\left[\pi_{1}, \pi_{4}\right]\left[\pi_{2}, \pi_{3}\right], \\
& \sum^{6}\left[\pi_{4}, \pi_{5}\right]\left[\pi_{1}, \pi_{2}, \pi_{3}\right]=\left[\pi_{1}, \pi_{4}\right]\left[\pi_{2}, \pi_{3}, \pi_{5}\right]+\left[\pi_{1}, \pi_{5}\right]\left[\pi_{2}, \pi_{3}, \pi_{4}\right]+\left[\pi_{2}, \pi_{4}\right]\left[\pi_{1}, \pi_{3}, \pi_{5}\right] \\
& +\left[\pi_{2}, \pi_{5}\right]\left[\pi_{1}, \pi_{3}, \pi_{4}\right]+\left[\pi_{3}, \pi_{4}\right]\left[\pi_{1}, \pi_{2}, \pi_{5}\right]+\left[\pi_{3}, \pi_{5}\right]\left[\pi_{1}, \pi_{2}, \pi_{4}\right]
\end{aligned}
$$

and

$$
\begin{aligned}
\sum^{15}\left[\pi_{1}, \pi_{2}\right]\left[\pi_{3}, \pi_{4}\right]\left[\pi_{5}, \pi_{6}\right]= & {\left[\pi_{1}, \pi_{2}\right]\left[\pi_{3}, \pi_{4}\right]\left[\pi_{5}, \pi_{6}\right]+\left[\pi_{1}, \pi_{3}\right]\left[\pi_{2}, \pi_{4}\right]\left[\pi_{5}, \pi_{6}\right] } \\
& +\left[\pi_{1}, \pi_{4}\right]\left[\pi_{2}, \pi_{3}\right]\left[\pi_{5}, \pi_{6}\right]+\left[\pi_{1}, \pi_{5}\right]\left[\pi_{2}, \pi_{3}\right]\left[\pi_{4}, \pi_{6}\right] \\
& +\left[\pi_{1}, \pi_{6}\right]\left[\pi_{2}, \pi_{3}\right]\left[\pi_{4}, \pi_{5}\right]+\left[\pi_{2}, \pi_{3}\right]\left[\pi_{1}, \pi_{4}\right]\left[\pi_{5}, \pi_{6}\right] \\
& +\left[\pi_{2}, \pi_{4}\right]\left[\pi_{1}, \pi_{3}\right]\left[\pi_{5}, \pi_{6}\right]+\left[\pi_{2}, \pi_{5}\right]\left[\pi_{1}, \pi_{3}\right]\left[\pi_{4}, \pi_{6}\right] \\
& +\left[\pi_{2}, \pi_{6}\right]\left[\pi_{1}, \pi_{3}\right]\left[\pi_{4}, \pi_{5}\right]+\left[\pi_{3}, \pi_{4}\right]\left[\pi_{1}, \pi_{2}\right]\left[\pi_{5}, \pi_{6}\right] \\
& +\left[\pi_{3}, \pi_{5}\right]\left[\pi_{1}, \pi_{2}\right]\left[\pi_{4}, \pi_{6}\right]+\left[\pi_{3}, \pi_{6}\right]\left[\pi_{1}, \pi_{2}\right]\left[\pi_{4}, \pi_{5}\right] \\
& +\left[\pi_{4}, \pi_{5}\right]\left[\pi_{1}, \pi_{2}\right]\left[\pi_{3}, \pi_{6}\right]+\left[\pi_{4}, \pi_{6}\right]\left[\pi_{1}, \pi_{2}\right]\left[\pi_{3}, \pi_{5}\right] \\
& +\left[\pi_{5}, \pi_{6}\right]\left[\pi_{1}, \pi_{2}\right]\left[\pi_{3}, \pi_{4}\right] .
\end{aligned}
$$

\section{Appendix B}

Theorem B.1. Suppose $X \sim \mathcal{C N}_{p}(0, V)$ so $V=E X X^{*}$. Its non-zero moments

$$
\mu_{1 \ldots n, n+1 \ldots 2 n}=E X_{1} \ldots X_{n} \bar{X}_{n+1} \ldots \bar{X}_{2 n}
$$

are $\mu_{1,2}=V_{12}, \mu_{12,34}=V_{13} V_{24}+V_{14} V_{23}$, and so on, satisfying the recurrence relation

$$
\mu_{1 \ldots n, n+1 \ldots 2 n}=\sum_{i=1}^{n} \mu_{1, n+i} \mu_{2 \ldots n,(n+1 \ldots 2 n)_{i}}
$$

where $(n+1 \ldots 2 n)_{i}$ drops the ith term. Similarly, for $i_{1}, \ldots, i_{n}, j_{1}, \ldots, j_{n}$ in $1, \ldots, p$,

$$
E X_{i_{1}} \ldots X_{i_{n}} \bar{X}_{j_{1}} \ldots \bar{X}_{j_{n}}=\sum^{n !} V_{i_{1} k_{1}} \ldots V_{i_{n} k_{n}}
$$

where $\sum^{n !}$ sums over all $n$ ! permutations $k_{1} \ldots k_{n}$ of $j_{1} \ldots j_{n}$.

So, in expanded form, the right hand side of (B.1) has $n$ ! terms, as compared with $1 \cdot 3 \ldots(2 n-1)=$ $(2 n) ! 2^{-n} / n !=E \mathcal{N}(0,1)^{2 n}$ terms for the real case $X \sim \mathcal{N}_{p}(0, V)$. Reed [6] proved (B.2) for the stationary case $V_{i j}$ a function of $i-j$, but his proof holds without this assumption. 
Theorem B.2. Let $q_{r}=X^{*} a_{r} X$ for $a_{r}$ in $\mathcal{C}^{p \times p}$. Set $B_{r}=a_{r} V$ and $t_{1 \cdots r}=\operatorname{trace}\left(B_{1} \cdots B_{r}\right)$. Then

$$
\begin{aligned}
E q_{1} & =t_{1}, E q_{1} q_{2}=t_{1} t_{2}+t_{12}, E q_{1} q_{2} q_{3}=t_{1} t_{2} t_{3}+\sum_{123}^{3} t_{1} t_{23}+\sum_{23}^{2} t_{123}, \\
E q_{1} \ldots q_{4} & =t_{1} t_{2} t_{3} t_{4}+\sum^{6} t_{1} t_{2} t_{34}+\sum^{4} t_{1} \sum_{34}^{2} t_{234}+\sum^{3} t_{12} t_{34}+\sum_{234}^{6} t_{1234},
\end{aligned}
$$

and in general

$$
E q_{1} \ldots q_{r}=\sum_{j=1}^{r} T_{r j} \text { for } T_{r j}=\sum_{\pi}\left\{t_{\pi_{1}} \ldots t_{\pi_{j}}:\left|\pi_{1}\right|+\ldots+\left|\pi_{j}\right|=r\right\}
$$

where for $\pi=\left(i_{1} \ldots i_{r}\right), t_{\pi}=\operatorname{trace}\left(B_{i_{1}} \ldots B_{i_{r}}\right)$, and $\sum_{\pi}$ sums over all such $\left(\pi_{1}, \ldots, \pi_{j}\right)$ giving different terms.

\section{Appendix C}

Theorem C.1 gives a closed expression for

$$
g_{M r}(\delta)=M^{-1} \sum_{m=0}^{M-1} \nu_{m}^{r} \exp \left(j \nu_{m} \delta\right),
$$

where $\nu_{m}=m / M-(M-1) / 2$.

Theorem C.1. Let $a=(M+1) /(2 M), b=(1-M) /(2 M), c=1 / M, N(x)=\exp (a x)-\exp (b x), D(x)=$ $\exp (c x)-1, I(x)=1 / D(x)$ and $G(x)=\sum_{m=0}^{M-1} \exp \left(x \nu_{m}\right)=N(x) I(x)$. We have

$$
g_{M r}(\delta)=M^{-1} G^{(r)}(j \delta)
$$

where

$$
G^{(r)}(x)=\sum_{k=0}^{r}\left(\begin{array}{l}
r \\
k
\end{array}\right) N_{r-k} c^{k} P_{k}, N_{i}=a^{i} \exp (a x)-b^{i} \exp (b x)
$$

and

$$
P_{k}=\sum_{i=0}^{k}(-1)^{i} i ! D^{-i-1} \exp (c i x) C_{k i}, C_{k i}=\sum_{n=0}^{i}\left(\begin{array}{l}
i \\
n
\end{array}\right)(-1)^{i-n} n^{k} .
$$

In particular,

$$
\begin{aligned}
& P_{0}=D^{-1}, P_{1}=D^{-1}-D^{-2} \exp (c x), P_{2}=D^{-1}-D^{-2} \exp (c x)+4 D^{-3} \exp (2 c x), \\
& P_{3}=D^{-1}-D^{-2} \exp (c x)+12 D^{-3} \exp (2 c x)-36 D^{-4} \exp (3 c x), \\
& P_{4}=D^{-1}-D^{-2} \exp (c x)+28 D^{-3} \exp (2 c x)-216 D^{-4} \exp (3 c x)+576 D^{-5} \exp (4 c x),
\end{aligned}
$$

and so on. Also

$$
g_{M r}(0)=M^{-1} \sum_{m=0}^{M-1} \nu_{m}^{r}=g_{r}
$$


say, is given by $g_{r}=0$ for $r$ odd, and for $d=M^{-2}$, by

$$
\begin{aligned}
g_{0} & =1, g_{2}=(1-d) / 12, g_{4}=\left(3-10 d+7 d^{2}\right) / 240, g_{6}=\left(3-21 d+49 d^{2}-31 d^{3}\right) / 1344 \\
g_{8} & =\left(5-60 d+294 d^{2}-620 d^{3}+381 d^{4}\right) / 11520 \\
g_{10} & =\left(3-55 d+462 d^{2}-2046 d^{3}+4191 d^{4}-2555 d^{5}\right) / 33792
\end{aligned}
$$

and so on.

\section{Appendix D}

Proof of Theorem 2.1. For $\left\{\Lambda_{N}\right\}$ sufficiently smooth, $\widehat{\theta}$ satisfies

$$
0=\Lambda_{\cdot 1}(\widehat{\theta})=\Lambda_{\cdot 1}+\Lambda_{\cdot{ }_{12}} \delta_{2} / 1 !+\Lambda_{\cdot 123} \delta_{2} \delta_{3} / 2 !+\ldots
$$

by the multivariate Taylor expansion, using the tensor summations convention: repeated pairs of suffixes are implicitly summed over their range $1 \ldots p$. For example, $\Lambda_{\cdot 12} \delta_{2}=\sum_{i_{2}=1}^{p} \Lambda_{.12} \delta_{2}$. So,

$$
0=\Lambda_{\cdot 1}+\left(A_{12}+\Delta_{12}\right) \delta_{2}+\left(A_{123}+\Delta_{123}\right) \delta_{2} \delta_{3} / 2+\ldots
$$

Multiply by $A^{01}$. Since $A^{01} A_{12}=1$ or 0 for $i_{0}=i_{2}$ or $i_{0} \neq i_{2}$, this gives

$$
0=\delta_{0}+A^{01}\left\{\Lambda_{\cdot 1}+\Delta_{12} \delta_{2}+\left(A_{123}+\Delta_{123}\right) \delta_{2} \delta_{3} / 2+\ldots\right\},
$$

where the first two terms have magnitude $O_{p}\left(n^{-1 / 2}\right)$, the next two are $O_{p}\left(n^{-1}\right)$ and so on. So, an expansion of the form (2.1) is possible. Substituting (2.1) into (D.1) gives $0=\sum_{a=1}^{\infty} T_{a}$, where $T_{a}=O_{p}\left(n^{-a / 2}\right)$ :

$$
\begin{aligned}
T_{1}= & \delta_{0}^{1}+A^{01} \Delta_{1}, T_{2}=\delta_{0}^{2}+A^{01}\left(\Delta_{12} \delta_{2}^{1}+A_{123} \delta_{2}^{1} \delta_{3}^{1} / 2\right), \\
T_{3}= & \delta_{0}^{3}+A^{01}\left(\Delta_{12} \delta_{2}^{2}+A_{123} \sum_{23}^{2} \delta_{2}^{1} \delta_{3}^{2}+\Delta_{123} \delta_{2}^{1} \delta_{3}^{1} / 2+A_{1 \ldots 4} \delta_{2}^{1} \delta_{3}^{1} \delta_{4}^{1} / 6\right), \\
T_{4}= & \delta_{0}^{4}+A^{01}\left\{\Delta_{12} \delta_{2}^{3}+A_{123}\left(\delta_{2}^{2} \delta_{3}^{2}+\sum_{23}^{2} \delta_{2}^{1} \delta_{3}^{3}\right) / 2+\Delta_{123} \sum_{23}^{2} \delta_{2}^{1} \delta_{3}^{2} / 2\right. \\
& \left.\left.+A_{1 \ldots 4} \sum_{234}^{3} \delta_{2}^{1} \delta_{3}^{1} \delta_{4}^{2} / 6+\Delta_{1 \ldots 4} \delta_{2}^{1} \delta_{3}^{1} \delta_{4}^{1} / 6+A_{1 \ldots 5} \delta_{2}^{1} \delta_{3}^{1} \delta_{4}^{1} \delta_{5}^{1} / 24\right)\right\},
\end{aligned}
$$

and so on. We can now obtain $\left\{\delta_{0}^{a}\right\}$ in (2.6)-(2.8) by setting $T_{a} \equiv 0$ and solving these recurrence relations for $\delta_{0}^{a}$.

Proof of Theorem 3.1. Note that $a_{12}^{11}=A^{13} A^{24} \kappa\left(\Delta_{3}, \Delta_{4}\right)$, so (3.7) follows. Note that $a_{0}^{1}=0$, so $a_{0.1}^{1}=0$, $a_{0}^{2}=A^{01} A^{23} E \Delta_{12} \Delta_{3}-B^{045} E \Delta_{4} \Delta_{5}$, so (3.8) follows. Note that $a_{123}^{111}=-A^{14} A^{25} A^{36} \kappa\left(\Delta_{4}, \Delta_{5}, \Delta_{6}\right)$, so $(3.9)$ follows. Note that

$$
a_{123}^{112}=A^{14} A^{25}\left\{A^{36} A^{78} \sum_{45}^{2} \kappa\left(\Delta_{4}, \Delta_{67}\right) \kappa\left(\Delta_{5}, \Delta_{8}\right)-B^{367} \sum_{45}^{2} \kappa\left(\Delta_{4}, \Delta_{6}\right) \kappa\left(\Delta_{5}, \Delta_{7}\right)\right\}+O\left(n^{-3}\right)
$$

since if

$$
E X_{i} \equiv 0 \text { and } \mu_{j_{1} \ldots j_{r}} \equiv E X_{j_{1}} \ldots X_{j_{r}}
$$


then

$$
\kappa\left(X_{1}, X_{2}, X_{3} X_{4}\right)=\kappa\left(X_{1}, X_{2}, X_{3}, X_{4}\right)+\sum_{12}^{2} \mu_{13} \mu_{24} .
$$

So, (3.10) follows. So, $k_{\cdot 2}^{123}$ is given by (3.4), (3.9) and (3.10). We next give $k_{\cdot 2}^{12}$ of (3.5). Recall that $a_{12 \cdot k}^{11}=0$ for $k \neq 1$. Note that

$$
a_{12}^{12}=-A^{13} \kappa\left(\Delta_{3}, A^{24} A^{56} \Delta_{45} \Delta_{6}-B^{245} \Delta_{4} \Delta_{5}\right)
$$

so (3.11) follows since (D.2) implies $\kappa\left(X_{1}, X_{2} X_{3}\right)=\mu_{123}$. Also

$$
\begin{aligned}
a_{12}^{13}= & A^{13} A^{24} \kappa\left(\Delta_{3}, \Delta_{45}\left(A^{56} A^{78} \Delta_{67} \Delta_{8}-B^{567} \Delta_{6} \Delta_{7}\right)\right. \\
& -2^{-1} A_{456} \sum_{56}^{2} A^{57} \Delta_{7}\left(A^{68} A^{9,10} \Delta_{89} \Delta_{10}-B^{689} \Delta_{8} \Delta_{9}\right) \\
& \left.\left.+2^{-1} A^{57} A^{68} \Delta_{456} \Delta_{7} \Delta_{8}-C_{4}^{567} \Delta_{5} \Delta_{6} \Delta_{7}\right)\right) \\
= & A^{13} A^{24}\left\{A^{56} A^{78} \kappa\left(\Delta_{3}, \Delta_{45} \Delta_{67} \Delta_{8}\right)-B^{567} \kappa\left(\Delta_{3}, \Delta_{45} \Delta_{6} \Delta_{7}\right)\right. \\
& -2^{-1} A_{456} \sum_{56}^{2} A^{57}\left(A^{68} A^{9,10} \kappa\left(\Delta_{3}, \Delta_{7} \Delta_{89} \Delta_{10}\right)-B^{689} \kappa\left(\Delta_{3}, \Delta_{7} \Delta_{8} \Delta_{9}\right)\right) \\
& \left.+2^{-1} A^{57} A^{68} \kappa\left(\Delta_{3}, \Delta_{456} \Delta_{7} \Delta_{8}\right)-C_{4}^{567} \kappa\left(\Delta_{3}, \Delta_{5} \Delta_{6} \Delta_{7}\right)\right\} .
\end{aligned}
$$

Also (D.2) implies

$$
\kappa\left(X_{1}, X_{2} X_{3} X_{4}\right)=\mu_{1 \ldots 4}=\kappa\left(X_{1}, \ldots, X_{4}\right)+\sum^{3} \mu_{12} \mu_{34} .
$$

So, (3.12) follows. Also

$$
\begin{aligned}
a_{12}^{22}= & \kappa\left(A^{13} A^{45} \Delta_{34} \Delta_{5}-B^{134} \Delta_{3} \Delta_{4}, A^{26} A^{78} \Delta_{67} \Delta_{8}-B^{267} \Delta_{6} \Delta_{7}\right) \\
= & A^{13} A^{45} A^{26} A^{78} \kappa\left(\Delta_{34} \Delta_{5}, \Delta_{67} \Delta_{8}\right) \\
& -\sum_{12}^{2} A^{13} A^{45} B^{267} \kappa\left(\Delta_{34} \Delta_{5}, \Delta_{6} \Delta_{7}\right)+B^{134} B^{267} \kappa\left(\Delta_{3} \Delta_{4}, \Delta_{6} \Delta_{7}\right) .
\end{aligned}
$$

Also (D.2) implies

$$
\kappa\left(X_{1} X_{2}, X_{3} X_{4}\right)=\mu_{1 \ldots 4}-\mu_{12} \mu_{34}=\kappa\left(X_{1}, \ldots, X_{4}\right)+\sum_{12}^{2} \mu_{13} \mu_{24} .
$$

So, (3.13) follows. So, $k_{.2}^{12}$ is given by (3.5), (3.11), (3.12), (3.13). Finally, we give $k_{.3}^{1234}$ starting from (3.6):

$$
\begin{aligned}
& a_{1234}^{1111}=A^{15} A^{26} A^{37} A^{48} \kappa\left(\Delta_{5}, \Delta_{6}, \Delta_{7}, \Delta_{8}\right), \\
& a_{1234}^{1112}=-A^{15} A^{26} A^{37} \kappa\left(\Delta_{5}, \Delta_{6}, \Delta_{7}, A^{48} A^{9,10} \Delta_{89} \Delta_{10}-B^{489} \Delta_{8} \Delta_{9}\right),
\end{aligned}
$$

so (3.14) follows. Also (D.2) implies

$$
\kappa\left(X_{1}, X_{2}, X_{3}, X_{4} X_{5}\right)=\kappa\left(X_{1}, \ldots, X_{5}\right)+\sum^{6} \mu_{45} \mu_{123} .
$$


So, (3.15) follows and

$$
\begin{aligned}
a_{1234}^{1113}= & A^{15} A^{26} A^{37} A^{48} \kappa\left(\Delta_{5}, \Delta_{6}, \Delta_{7}, \Delta_{89}\left(A^{9,10} A^{11,12} \Delta_{10,11} \Delta_{12}-B^{9,10,11} \Delta_{10} \Delta_{11}\right)\right. \\
& -2^{-1} A_{89,10} \sum_{9,10}^{2} A^{9,11} \Delta_{11}\left(A^{10,12} A^{13,14} \Delta_{12,13} \Delta_{14}-B^{10,12,13} \Delta_{12} \Delta_{13}\right) \\
& \left.+2^{-1} A^{9,11} A^{10,12} \Delta_{89,10} \Delta_{11} \Delta_{12}-C_{8}^{9,10,11} \Delta_{9} \Delta_{10} \Delta_{11}\right) .
\end{aligned}
$$

Also setting $k_{1 \ldots r}=\kappa\left(X_{1}, \ldots, X_{r}\right),($ D.2) implies

$$
\begin{aligned}
\kappa\left(X_{1}, X_{2}, X_{3}, X_{4} X_{5} X_{6}\right)= & \mu_{1 \ldots 6}-\sum_{123}^{3} \mu_{12} \mu_{3 \ldots 6}=k_{1 \ldots 6}+\sum^{10} k_{123} k_{456}+\sum^{15} \mu_{12} \mu_{34} \mu_{56} \\
& +\sum^{12} \mu_{14} k_{2356} .
\end{aligned}
$$

So, (3.16) follows and

$$
a_{1234}^{1122}=A^{15} A^{26} \kappa\left(\Delta_{5}, \Delta_{6}, A^{37} A^{89} \Delta_{78} \Delta_{9}-B^{378} \Delta_{7} \Delta_{8}, A^{4,10} A^{11,12} \Delta_{10,11} \Delta_{12}-B^{4,10,11} \Delta_{10} \Delta_{11}\right) .
$$

Also (D.2) implies

$$
\kappa\left(X_{1}, X_{2}, X_{3} X_{4}, X_{5} X_{6}\right)=\underset{\substack{1 \ldots 6 \\-\mu_{134} \mu_{256}-\mu_{234} \mu_{156} .}}{k_{15}} \mu_{12} k_{3 \ldots 6}+\sum_{123}^{10} \mu_{456}+\sum^{15} \mu_{12} \mu_{34} \mu_{56}
$$

So, (3.17) follows. So, $k_{.3}^{1234}$ is given by $(3.6),(3.14)-(3.17)$.

Proof of Theorems 4.1 and 4.2. Note that $A_{1}=0$ as required. Also

$$
\begin{aligned}
A_{12} & =[12]=n^{-1} \sum_{N=1}^{n} S_{N \cdot 1} S_{N \cdot 2}=\langle 1,2\rangle, \\
A_{123} & =[123]=\sum^{3}\langle 1,23\rangle, \\
A_{1234} & =[1234]=\sum^{3}\langle 12,34\rangle+\sum^{4}\langle 1,234\rangle, \\
{\left[\pi_{1}, \ldots, \pi_{r}\right] } & =(-1)^{r} \lambda_{r}\left\langle\pi_{1}, \ldots, \pi_{r}\right\rangle
\end{aligned}
$$

for $r \geq 1$. So, $[1,2]=\lambda_{2}\langle 1,2\rangle=\lambda_{2} A_{12}$. So, by (3.7), the asymptotic covariance of $\widehat{\theta}$ is given by (4.4). By (3.8), the asymptotic bias of $\widehat{\theta}$ is given by (4.5). For $A$ diagonal (4.5) reduces to (4.14). By (3.4), (3.9) and (3.10), we have (4.6) and (4.15). Note that $k_{.2}^{12}$ is given by (3.5) in terms of (4.7)-(4.9). For $A$ diagonal, (4.7), (4.8) and (4.9) reduce to $(4.16),(4.17)$ and $(4.18)$, respectively. Note that $k_{3}^{i_{1} i_{2} i_{3} i_{4}}=k_{\cdot 3}^{1234}$ is given by (3.6) in terms of (4.10)-(4.13). For $A$ diagonal, (4.10)-(4.13) reduce to (4.19)-(4.22).

Proof of Theorem 6.1. Using the notational conventions of Section 2, $\operatorname{tr} Q=n-q$ so $\operatorname{tr} Q \cdot 1 \ldots r=0$ for $r \geq 1$. Also $\Lambda_{\cdot 1 \ldots r}=n^{-1} Y^{\prime} Q_{\cdot 1 \ldots r} Y$, so

$$
A_{1 \ldots r}=n^{-1} \operatorname{tr} Q \cdot 1 \ldots r\left(\lambda_{2} I_{n}+S S^{\prime}\right)=n^{-1} S^{\prime} Q_{\cdot 1 \ldots r} S=n^{-1} \beta^{\prime} X^{\prime} Q_{\cdot 1 \ldots r} X \beta
$$


Also

$$
\begin{aligned}
& P X=X, Q X=0, Q \cdot{ }_{1} X+Q X_{\cdot 1}=0, X^{\prime} Q \cdot 1=-X^{\prime} Q X_{\cdot 1}=0, A_{1}=0 \\
& Q \cdot 12 X+Q \cdot 1 X_{\cdot 2}+Q \cdot{ }_{\cdot 2} X \cdot{ }_{\cdot 1}+Q X_{\cdot 12}=0 \\
& X^{\prime} Q \cdot 12 X=-\sum_{12}^{2} X^{\prime} Q \cdot{ }_{\cdot 1} X_{\cdot 2}=\sum_{12}^{2} X_{\cdot 1}^{\prime} Q X_{\cdot 2} \\
& Q \cdot 123 X+\sum_{123}^{3}\left(Q \cdot 12 X_{\cdot 3}+Q \cdot 3 X_{\cdot 12}\right)+Q X_{\cdot 123}=0 \\
& X^{\prime} Q \cdot 123 X=-\sum_{123}^{3} X^{\prime}\left(Q \cdot 12 X_{\cdot 3}+Q \cdot{ }_{\cdot 3} X_{\cdot 12}\right)=\sum_{123}^{6} X_{\cdot 1}^{\prime} Q \cdot{ }_{\cdot 2} X_{\cdot 3}+\sum_{123}^{3} X_{\cdot 1}^{\prime} Q X_{\cdot 23} .
\end{aligned}
$$

By (6.2),

$$
n^{-1} X^{\prime} X=n^{-1} \sum_{N=1}^{n} x_{N} x_{N}^{\prime}=T+O\left(n^{-1}\right)
$$

as $n \rightarrow \infty$, where $T=\int_{0}^{1} y(t) y(t)^{\prime} \mathrm{d} t$. (Higher order terms are given by the Euler-McLaurin expansion.) Similarly, for $\pi_{1}, \pi_{2}$ sequences of integers,

$$
n^{-1} X_{\cdot \pi_{1}}^{\prime} X_{\cdot \pi_{2}}=n^{-1} \sum_{N=1}^{n} x_{N \cdot \pi_{1}} x_{N \cdot \pi_{2}}^{\prime}=T\left(\pi_{1}, \pi_{2}\right)+O\left(n^{-1}\right) .
$$

Also

$$
n^{-1} X_{\cdot \pi_{1}}^{\prime} P X_{\cdot_{2}}=\left(n^{-1} X^{\prime} X_{\pi_{1}}\right)^{\prime}\left(n^{-1} X^{\prime} X\right)^{-1}\left(n^{-1} X^{\prime} X_{\pi_{2}}\right)=T\left(\cdot, \pi_{1}\right)^{\prime} T^{-1} T\left(\cdot, \pi_{2}\right)+O\left(n^{-1}\right),
$$

where $T\left(\cdot, \pi_{2}\right)$ is $T\left(\pi_{1}, \pi_{2}\right)$ with $\pi_{1}$ empty. So,

$$
n^{-1} X_{\pi_{1}}^{\prime} Q X_{\pi_{2}}=T\left(\pi_{1}: \pi_{2}\right)+O\left(n^{-1}\right) .
$$

So, by (D.7), $n^{-1} X^{\prime} Q_{12} X$ is bounded. So, by (D.6) $A_{12}$ is bounded. The condition that $A=\left(A_{12}\right)$ be bounded away from zero holds because of (6.3). Similarly, the second term in the right hand side of (D.8) is $O(n)$. That the first term is also follows similarly from

$$
Q \cdot 1=-P_{\cdot 1}=-X{ }_{\cdot 1} M X^{\prime}-X M_{\cdot 1} X^{\prime}-X M X_{\cdot 1}^{\prime} \text { and } M_{\cdot 1}=-M L \cdot{ }_{1} M
$$

where $M^{-1}=L=X^{\prime} X$. So, $A_{123}$ is bounded. Similarly, $A_{1 \ldots r}$ is bounded.

We now show that $\Delta_{\pi}=O_{p}\left(n^{-1 / 2}\right)$. Note that $\Delta_{\pi}=2 \Delta_{1 \pi}+\Delta_{2 \pi}$ for $\Delta_{1 \pi}=n^{-1} e^{\prime} Q \cdot \pi S$ and $\Delta_{2 \pi}=$ $n^{-1} e^{\prime} Q_{\cdot \pi} e$. Also $\Delta_{1 \pi} \dot{\sim} \mathcal{N}\left(0, n^{-1} \lambda_{2} \beta^{\prime} V_{n} \beta\right)$ for $V_{n}=n^{-1} X^{\prime} Q_{\cdot \pi}^{2} X=O(1)$ by a similar argument to above. So, $\Delta_{1 \pi}=O_{p}\left(n^{-1 / 2}\right)$. Also

$$
E \Delta_{2 \pi}=n^{-1} \lambda_{2} \operatorname{tr} Q_{\cdot \pi}=0, n^{2} E \Delta_{2 \pi}^{2}=\lambda_{4} \sum_{N=1}^{n} Q_{N N \cdot \pi}^{2}+2 \lambda_{2}^{2} \operatorname{tr} Q_{\cdot \pi}^{2},
$$

where $Q_{N_{1} N_{2}}^{2}$ are the elements of $Q$. By a similar argument to above

$$
\sum_{N=1}^{n} Q_{N N \cdot \pi}^{2}=O\left(n^{-1}\right) \text { and } \operatorname{tr} Q_{\cdot \pi}^{2}=O(1)
$$


So, $\Delta_{2 \pi}=O_{p}\left(n^{-1}\right)$. So, the conditions needed for Section 2 hold.

Proof of Theorem C.1. Note that (C.1) holds. By Leibniz' rule,

$$
G^{(r)}(x)=\sum_{k=0}^{r}\left(\begin{array}{l}
r \\
k
\end{array}\right) N^{(r-k)}(x) I^{(k)}(x) .
$$

Also note that $N^{(i)}(x)=N_{i}$. By Faa di Bruno's rule, Comtet ([2], page 137), for $f(D)=D^{-1}$,

$$
I^{(k)}(x)=\sum_{i=0}^{k} f_{i} B_{k i}(y)
$$

for $f_{i}=f^{(i)}(D)=(-1)^{i} i ! D^{-i-1}, y_{i}=D^{(i)}(x)=c^{i} \exp (c x)-\delta_{i 0}$, and $B_{k i}(y)$ the partial exponential Bell polynomial tabled on page 307 of [2]. These are defined in terms of

$$
S=\sum_{k=1}^{\infty} z^{k} y_{k} / k !
$$

by

$$
S^{i} / i !=\sum_{k=i}^{\infty} z^{k} B_{k i}(y) / k !
$$

But in our case $S=\exp (c x)\{\exp (c z)-1\}$ giving $B_{k i}(y)=\exp (i c x) c^{k} C_{k i} / i$ !. As an aside, since $B_{k k}(y)=y_{1}^{k}$, we obtain the interesting identity $C_{k i}=0$ for $k<i, C_{k k}=k$ !. So, with (C.1) we obtain the results of the theorem.

Acknowledgements. The authors would like to thank the Editors-in-Chief, the Associate Editor and the referee for carefully reading the paper and for their comments which greatly improved the paper.

\section{REFERENCES}

[1] J.G. Booth, P. Hall and A.T.A. Wood, On the validity of Edgeworth and saddlepoint approximations. Journal of Multivariate Analysis 51 (1994) 121-138.

[2] L. Comtet, Advanced Combinatorics. Reidel, Dordrecht, Holland (1974).

[3] R. Gatto and E. Ronchetti, General saddlepoint approximations of marginal densities and tail probabilities. Journal of the American Statistical Association 91 (1996) 666-673.

[4] Y. Kakizawa and M. Taniguchi, Higher order asymptotic relation between Edgeworth approximation and saddlepoint approximation. Journal of the Japan Statistical Society 24 (1994) 109-119.

[5] A.C. Monti, A new look at the relationship between Edgeworth expansion and saddlepoint approximation. Statistics and Probability Letters 17 (1993) 49-52.

[6] I.S. Reed, On a moment theorem for complex Gaussian processes. IRE Transactions on information theory IT-8 (1962) 194-195

[7] C.S. Withers and S. Nadrajah, The bias and skewness of (univariate) $M$-estimates in regression. Technical Report, Applied Mathematics Group, Industrial Research Ltd., Lower Hutt, New Zealand (2007).

[8] C.S. Withers and S. Nadarajah, Tilted Edgeworth expansions for asymptotically normal vectors. Annals of the Institute of Statistical Mathematics, doi: 10.1007/s10463-008-0206-0 (2008). 